\title{
Development of Novel Sorbents for Uranium Extraction from Seawater
}

\section{Fuel Cycle R\&D}

Dr. Wenbin Lin University of Chicago

In collaboration with:

Savannah River National Laboratory

Stephen Kung, Federal POC Sheng Dai, Technical POC

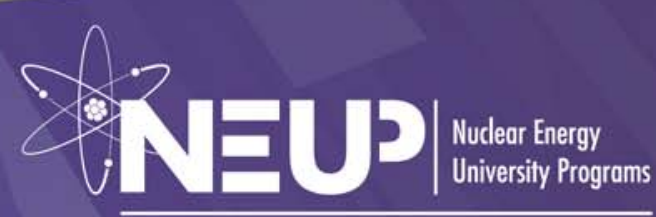


NEUP 11-3151

\title{
Development of Novel Sorbents for Uranium Extraction from Seawater
}

\author{
PI: Wenbin Lin, University of Chicago \\ Collaborator: Kathryn Taylor-Pashow, Savannah River National Lab \\ Technical Work Scope Identifier No.: 11-1799 Fuel Cycle R\&D (MS-FC)
}

\section{SUMMARY:}

The objective of this project was to develop novel sorbents for the extraction of uranium from seawater, with the goal of taking advantage of recent breakthroughs in nanoscience and nanotechnology to incorporate high densities of well-designed chelators into nanoporous supports to allow selective and efficient binding of uranyl ions from seawater. It was anticipated the proposed nanostructured sorbent materials would demonstrate higher binding capacity, enhanced extraction kinetics, optimal stripping efficiency for uranyl ions, and improved mechanical and chemical stabilities compared to traditional amidoxime-functionalized polymeric fibers. In addition to expanding the current state of knowledge for uranium extraction from seawater, potential was expected for translation of developed technologies to applications in environmental remediation and the enrichment and sequestration of ultralow concentrations of other metals.

The proposed research involved nine stated objectives: (1) Amidoxime-containing mesoporous silica nanoparticles (MSNs) were to be synthesized and characterized. (2) Initial sorption studies with depleted uranium solutions were to be performed using these MSN sorbents. (3) Detailed uranyl sorption studies regarding sorbent selectivity and kinetics were to be performed upon promising MSN sorbents identified during initial screenings. (4) Further optimization of these materials was to be performed, based off results from sorption studies. (5) Development of other nanoporous supports, including mesoporous carbon (MCNs) and metal-organic frameworks (MOFs) was to be initiated following preliminary studies with MSNs. (6) Uranyl sorption, sorbent selectivity, and kinetic studies would be performed on both MCN and MOF materials. (7) The effects of chelating amidoxime ligands on uranyl extraction were to be studied through combination of sorption data and computation. (8) The most promising nanostructured sorbents were to be identified. (9) Collaborative endeavors were to be identified and initiated to develop nanocomposite materials for environmental seawater studies.

A series of new MSN sorbents functionalized with amidoxime-, imide dioxime-, phosphonate-, and carboxylate-functional groups were synthesized, characterized, and investigated for extraction of uranium from water and artificial seawater at $\mathrm{pH}$ 8.3. All sorbents exhibited a high sorption capacity in water, with Langmuir isotherms indicating a saturation capacity as high as $185 \mathrm{mg} / \mathrm{g}$ for the best material. U sorption in artificial seawater is reduced fourfold, with a maximum uranium sorption of $12.1 \mathrm{mg} / \mathrm{g}$ obtained. This loading capacity is comparable with that obtained for amidoxime fibers under similar conditions. Fitting the sorption isotherms identified a diethoxyphosphonate and a cyclic imide dioxime as the best sorbents with the highest saturation capacities and greatest binding affinities.

A complementary series of organic sorbent ligands were used to functionalize a MCN template and applied to sorption of uranium from aqueous media under two different conditions. Ligands synthesized for grafting were previously reported to have affinity for uranium, and could be broadly categorized as amidoxime-, carboxyl- or phosphoryl-derived. The unfunctionalized MC material is known to have no background sorption, providing the first head-to-head comparison of uranyl-binding sorbent groups on an identical innocent support. Analysis of uranium sorption from acidic water and seawater 
simulant revealed several sorbents meriting further investigation. A detailed analysis of sorption by MC$\mathrm{O}-\mathrm{PO}(\mathrm{OH})_{2}$, obtained through phosphorylation of the mesoporous carbon support, revealed significant $\mathrm{pH}$ dependence where sorption significantly increased with an inflection point approximately equal to the $\mathrm{pk}_{\mathrm{a}}$ of the sorbent group. Adsorption isotherms yielded maximum saturation at $97 \mathrm{mg}$ of $\mathrm{U} / \mathrm{g}$ of sorbent in acidic water and $67 \mathrm{mg}$ of uranium/g of sorbent according to the Langmuir model. Kinetic analysis revealed rapid uranium sorption in seawater simulant, with more gradual sorption in acidic water, ultimately yielding a higher sorption. This study demonstrates MCNs judiciously functionalized with a sorbent ligand have great potential for uranium sorption.

Two porous metal-organic frameworks were prepared, characterized, and the first ever applied for actinide extraction. A MOF functionalized with a diethoxyphosphoryl-urea moiety exhibited near quantitative removal of uranium from water and artificial seawater, surpassing that of amidoxime polymers by at least four-fold. The aforementioned MOF has a saturation sorption capacity of $217 \mathrm{mg} \mathrm{U}$ / g sorbent, corresponding to binding of one uranyl ion for every two phosphoryl urea groups. DFT calculations indicate a monodentate coordination motif where the $\mathrm{UO}_{2}{ }^{2+}$ cation interacts with the phosphoryl oxygen on the functional species, with two ligands binding to each $\mathrm{UO}_{2}{ }^{2+}$ in the MOF. The sorbent groups are directed toward the center of the tetrahedral cavities in the MOF, and are properly orientated to enable cooperative binding and create a coordination pocket for $\mathrm{UO}_{2}{ }^{2+}$. These results indicate that porous MOF materials with phosphorylurea functional groups are good candidates for $U$ sorption under acidic conditions, and represent promising alternatives to organic polymer sorbents in $U$ extraction from nuclear waste and acid mine drainage.

Theoretical investigations of uranyl-amidoxime complexes were performed in gas phase and with solvation modeling. Investigation of electronic structure revealed similarities to uranyl-peroxide complexes, with major bonding contributions coming from the oxygen lone pair interacting with the uranyl $\sigma(\mathrm{f})$, as well as other interactions with the $\mathrm{N}-\mathrm{O} \pi$ orbitals. A series of uranyl-amidoxime derivatives were investigated to examine the role of electron donation on uranyl bond strength. Plotting the $\log \left(K / K_{0}\right)$ vs. the Hammett $\sigma_{\text {para }}$ value for the adjacent substituent revealed a linear relationship. The distance from uranium to the oxime function decreased as electron donation increased, as did the $\mathrm{O}=\mathrm{U}=\mathrm{O}$ bond angle and $\mathrm{U}=\mathrm{O}$ bond index. Electron donation was further increased by attaching the oxime function to an imidazole or imidazolidin rings, giving the most thermodynamically favored uranyl complexes investigated in this study. Application of these findings to the development of new sorbents for uranyl extraction demonstrate the utility of adjacent Lewis bases, such as peroxide or oxime functions, and reveal the necessity of maximizing electron density at the binding site through preservation of conjugated $\pi$ orbitals and electron donation via resonance.

Several promising sorbent groups have been identified for uranyl sorption. Surprisingly, amidoxime-derived sorbents were inferior to a phosphate-derived material on both mesoporous supports, suggesting the $\mathrm{KOH}$ treatment process changes the sorption chemistry routinely attributed to amidoxime. The diethoxyphosphorylurea functional group was observed to be very competitive when oriented in a fashion to facilitate cooperative interactions with the same uranium atom.

Phosphonate-functionalized mesoporous carbon materials have been sent to Pacific Northwest National Lab for marine toxicology testing and also to Oak Ridge National Lab for confirmation of initial seawater testing results. Current engineering limitations prevent the testing of nanomaterials in a customary seawater flow-through apparatus at PNNL, but efforts are ongoing to develop monolithic materials or external supports capable of facilitating seawater testing of these newly-developed nanosorbents. 
NEUP 11-3151

\section{FULL STATEMENT OF EACH OBJECTIVE AND DESCRIPTION OF THE EFFORT PERFORMED AND ACCOMPLISHMENTS ACHIEVED}

\section{Synthesis and characterization of amidoxime-containing MSNs}

Three amidoxime-functionalized organic ligands and one imide dioxime ligand were prepared for grafting into MSNs (Figure 1). The amidoxime-based materials were produced using two different methods. In the first approach, the sorbent material was syntheized by grafting the corresponding nitrile silane derivative onto MSU-H, followed by conversion of the nitrile to an amidoxime group by treatment with hydroxylamine (MSA-I, MSA-3). The second approach involved synthesis of the amidoxime and imide dioxime compounds, followed by reaction with trialkoxysilane to form the silane derivative for grafting onto MSU-H (MSA-II, MSCA-I).

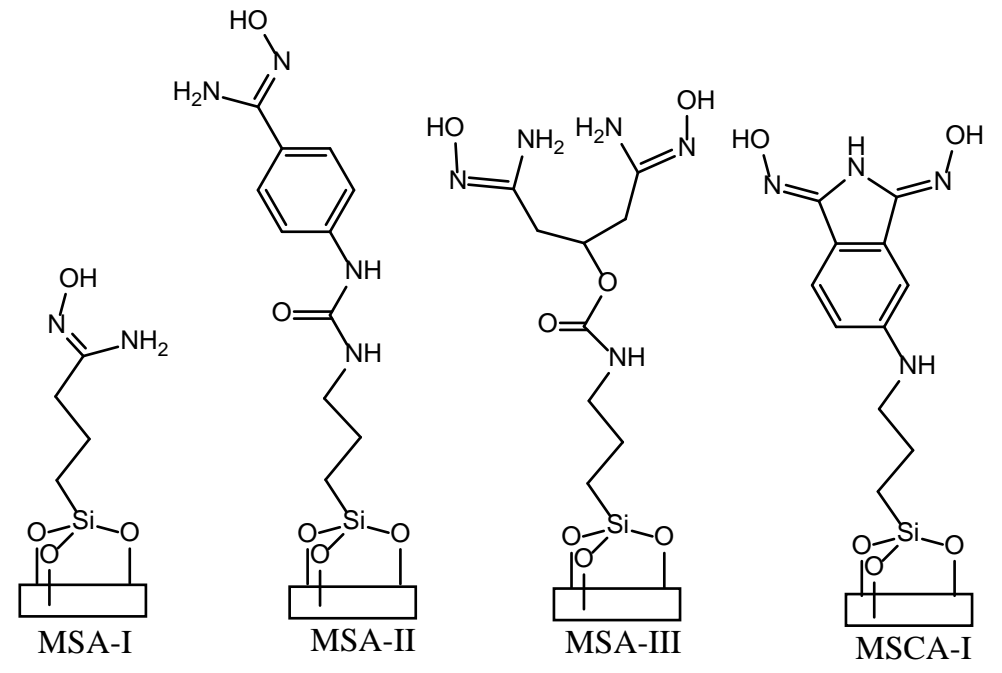

Figure 1. Amidoxime-derived organic ligands as grafted onto MSNs.

The structural and chemical properties of these sorbents were characterized by nitrogen adsorption (surface areas an pore diameters), $\zeta$-potential, IR spectroscopy, and thermogravimetric analysis. Characterization data are summarized in Table 1. TGA results indicate grafting densities of 0.75 to $1.32 \mathrm{mmol} / \mathrm{g}$. Nitrogen sorption isotherms are type IV for all sorbents, which are typical of mesoporous materials. The Brunauer-Emmett-Teller (BET) surface areas range from 186 to $526 \mathrm{~m}^{2} / \mathrm{g}$, with average pore diameters ranging from 3.8 to $7.8 \mathrm{~nm}$. The surface areas and average pore diameters for the functionalized MS materials are smaller than those of the unfunctionalized MSU-H, which has a surface area of $648.3 \mathrm{~m}^{2} / \mathrm{g}$ and average pore size of $9.6 \mathrm{~nm}$, consistent with successful functionalization of the MSU-H support and retention of material porosity. Analysis of these isotherms reveals minimal tailing in the mesoporous region, and indicate homogeneous grafting on the silica support. Similar sorbent loading between all materials suggest thorough surface area coverage.

FT-IR spectra of the all functionalized materials exhibited characteristic vibrations corresponding to $\mathrm{Si}-\mathrm{O}-\mathrm{Si}$, Si-C, and Si-O-C stretching. Amidoxime-functionalized materials also possessed several diagnostic bands, such as a broad peak around 3600-3300 associated with $\mathrm{O}-\mathrm{H}, \mathrm{N}-\mathrm{H}$, free oxime, and oxime stretching, as well as a peak at $1671 \mathrm{~cm}^{-1}$ due to hydroxyimine, characteristic of the amidoxime functional group. The imide dioxime material (MSCA-I) showed a vibration peak at $3025.6 \mathrm{~cm}^{-1}$, corresponding to the $\mathrm{C}-\mathrm{H}$ stretch in the aromatic group. These data confirm the uranium-binding functionality is preserved during the grafting process. 
Surface charge of the amidoxime-functionalized materials was measured as $\zeta$-potential by dynamic light scattering measurements. The $\zeta$-potential varied as a function of the moiety grafted onto the silica support. The $\zeta$-potential shifted from $-27.7 \mathrm{mV}$ for unfunctionalized MSU-H to increasingly positive values after functionalization $(-10.2 \mathrm{mV},+15.2 \mathrm{mV},+26.8 \mathrm{mV}$, and $+11.4 \mathrm{mV}$ for MSA-I, -II, III, and MSCA-I, respectively).

Table 1. Structural properties of amidoxime-derived mesoporous silica materials

\begin{tabular}{|l|c|c|c|c|}
\hline Material & $\begin{array}{l}\text { BET surface } \\
\text { area }\left(\mathrm{m}^{2} / \mathrm{g}\right)\end{array}$ & $\begin{array}{l}\text { BJH pore } \\
\text { size }(\mathrm{nm})\end{array}$ & $\begin{array}{l}\text { Loading } \\
(\mathrm{mmol} / \mathrm{g})\end{array}$ & $\zeta$-potential $(\mathrm{mV})$ \\
\hline MSU-H & 648 & 9.6 & --- & $-27.7 \pm 1.3$ \\
\hline MSA-I & 494 & 7.8 & 0.75 & $-10.2 \pm 2.0$ \\
\hline MSA-II & 186 & 4.9 & 1.09 & $+15.1 \pm 0.7$ \\
\hline MSA-III & 526 & 7.8 & 1.02 & $+26.8 \pm 1.5$ \\
\hline MSCA-I & 277 & 5.6 & 1.32 & $+11.4 \pm 0.2$ \\
\hline
\end{tabular}

\section{Initial Sorption Studies with depleted uranium solutions}

The grafting of these organic functionalities onto an identical commercially available silica support was intended to create materials with similar loading, porosity, and surface area. The resulting differences in sorption capacity could then be attributed to different affinities of the organic moieties, rather than the physical properties of the support. The unfunctionalized MSU-H was investigated for comparison, as this material is known to bind uranium through a non-specific sorption mechanism. While non-functionalized space on the silica materials physisorb uranium, all materials showed similar grafting density and homogeneous functional group distribution, suggesting the extent of physisorption to be consistent for all materials.

An aqueous solution was prepared with uranium concentration of $5 \mathrm{ppm}$, facilitating rapid screening experiments and ease of analysis by UV-visible spectroscopy. Solution pH was adjusted to 8.3 by adding small volumes of $1 \mathrm{M}$ sodium hydroxide. Sorbents were added to aqueous solutions at a phase ratio of $0.1 \mathrm{mg} / \mathrm{mL}$ and agitated at $300 \mathrm{rpm}$ on an orbital shaker for $1 \mathrm{hr}$ at room temperature. Sorbents were then separated by centrifugation and uranium concentration in the supernatant determined by UVvisible spectroscopy with the Arsenazo (III) assay. Concentrations were determined against a standard curve, with a blank and uranium-spiked water without sorbents analyzed as controls.

Amidoxime-functionalized MS sorbents showed a high uranyl sorption capacity at equilibrium $\left(q_{e}\right)$, ranging from 40 to $50 \mathrm{mg}$ uranium $/ \mathrm{mg}$ sorbent. The difference between sorbents under these experimental conditions was not statistically significant, but the high uranium extraction revealed all amidoxime-functionalized materials showed great promise as sorbents. Figure 2 displays the sorption data for all functionalized mesoporous silica materials in water.

Uranium absorbed by the functionalized materials was eluted by successive washing with $10 \mathrm{~mL}$ of $0.01 \mathrm{M}, 0.1 \mathrm{M}$, and $1.0 \mathrm{M} \mathrm{HCl}$ aqueous solutions. Each wash solution was then analyzed by UV-visible spectroscopy, as described above. The total amount of uranyl eluted from the MS sorbents was the sum of quantities measured in the three eluent solutions. These studies revealed that relatively weak $\mathrm{HCl}$ $(0.1 \mathrm{M})$ elutes virtually all uranium from most sorbent materials, $1.0 \mathrm{M} \mathrm{HCl}$ was required to desorb the significant fraction of uranium that remained in MSCA-I after $0.1 \mathrm{M} \mathrm{HCl}$ elution. Table 2 shows all elution data for functionalized mesoporous-silica sorbents. 


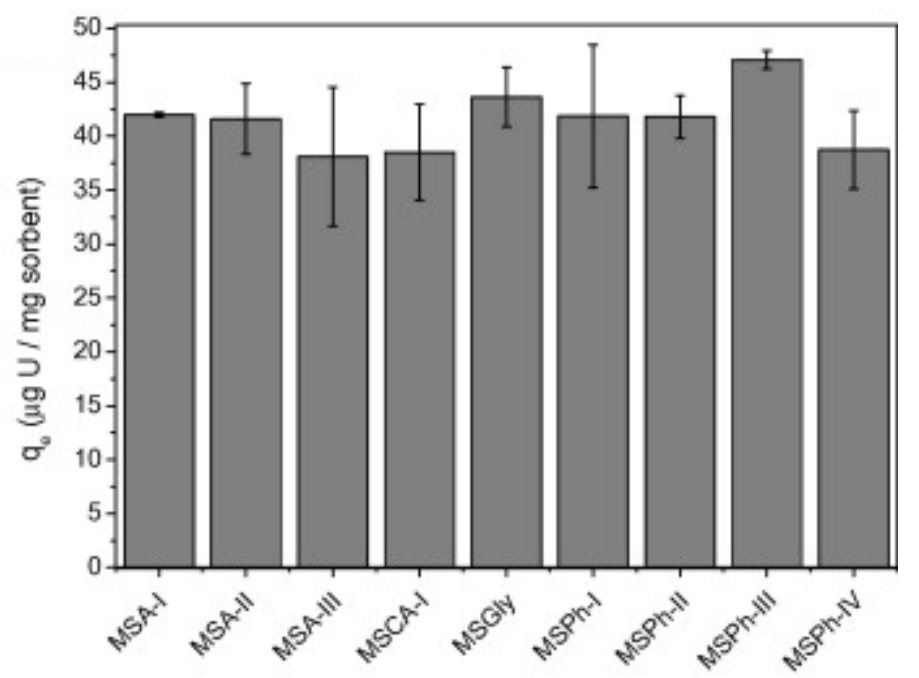

Figure 2. Comparison of uranium sorption capacities for different organo-functionalized mesoporous silica materials in water. $\mathrm{pH} 8+/-0.1 ; m_{\text {sorbent }} / v_{\text {solution }}=0.1 \mathrm{mg} / \mathrm{mL} ;[\mathrm{U}]_{\text {initial }}=5 \mathrm{ppm}$.

Table 2. Elution of uranium from mesoporous silica sorbents (Efficiency, \%)

\begin{tabular}{|c|c|c|c|}
\hline Sample & $\mathbf{0 . 0 1} \mathbf{~ M ~ H C l}$ & $\mathbf{0 . 1} \mathbf{~ M ~ H C l}$ & $\mathbf{1 . 0}$ M HCl \\
\hline MSU-H & 63.3 & $>99$ & $>99$ \\
\hline MSA-I & 63.7 & $>99$ & $>99$ \\
\hline MSCA-I & 29.1 & 95.7 & $>99$ \\
\hline MSPh-II & 24.0 & 98.7 & $>99$ \\
\hline MSPh-III & 16.9 & 79.9 & $>99$ \\
\hline
\end{tabular}

Detailed uranyl sorption selectivity and kinetics studies on MSNs

The amidoxime-functionalized MSN materials were investigated for extraction of uranium from seawater simulant to investigate the effects of carbonate and salts on sorption. No competing metals were included for these preliminary tests, but will be included in future investigations. Artificial seawater was prepared as reported in the literature with an initial uranium concentration of $5 \mathrm{ppm}$. After sorption experiments, described above, uranium concentration was measured by UV-visible spectroscopy as discussed previously.

Extraction of uranium from artificial seawater was reduced at least fourfold compared to the initial studies performed in water (Figure 3). For the amidoxime-functionalized materials, sorption capacities of approximately $5-7 \mathrm{mg} / \mathrm{g}$ were obtained, though the differences were not statistically significant. The presence of carbonates in solution reduces sorption, presumably due to the formation of uranium-triscarbonato complexes which do not bind as strongly. These materials were competitive with sorption exhibited under laboratory conditions by amidoxime-functionalized polymeric braids (6.0 mg/g). In contrast to the amidoxime fiber, amidoxime-functionalized MS materials did not receive a $\mathrm{KOH}$ pretreatment prior to sorption measurements. While such pretreatments are known to enhance extraction of uranium, it has been suggested they alter the actual sorbent group, forming a different binding ligand with improved sorption properties. In an effort to avoid such ambiguity, $\mathrm{KOH}$ pretreatments were not applied to the amidoxime-functionalized materials at any point. 
NEUP 11-3151

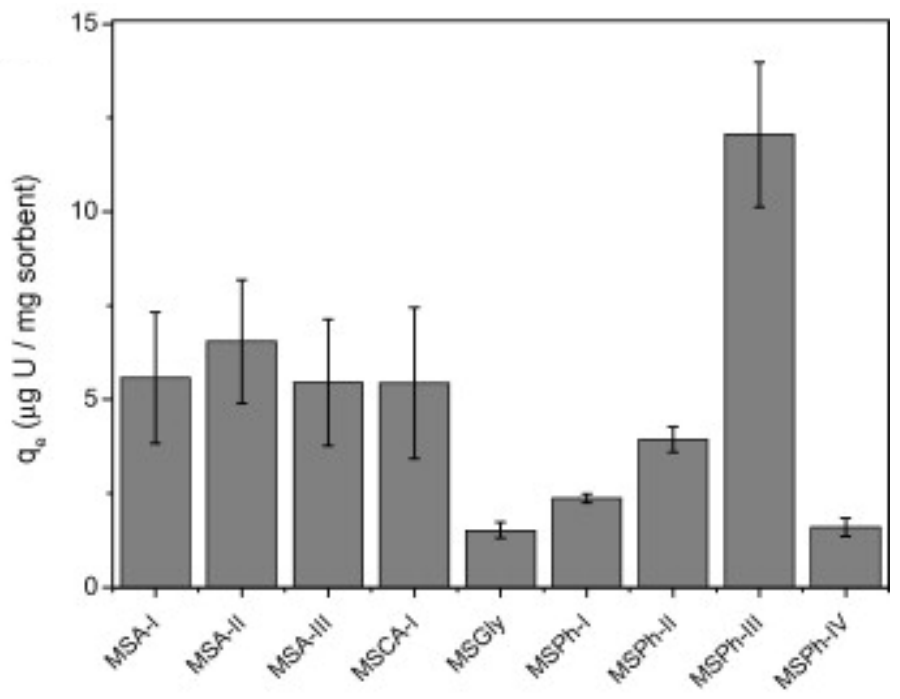

Figure 3. Comparison of uranium sorption capacities for different organo-functionalized mesoporous silica materials in seawater simulant. $\mathrm{pH} 8.3+/-0.1 ; m_{\text {sorbent }} / v_{\text {solution }}=0.1 \mathrm{mg} / \mathrm{mL} ;[\mathrm{U}]_{\text {initial }}=5 \mathrm{ppm}$.

Langmuir sorption isotherms were obtained for three of the amidoxime-functionalized materials (MSA-II, MSA-III, MSCA-I) by slight modification of the protocol for typical sorption experiments. Water and artificial seawater were prepared with varied concentrations of $U(1,5,10,20,30$ and 40 ppm in water; $1,4,8,17,25$, and 33 in seawater), with specific uranium concentrations determined prior to the experiment. MS sorbent was added at a phase ratio of $0.1 \mathrm{mg} / \mathrm{mL}$, and the suspension was then shaken for 1 hour at $300 \mathrm{rpm}$ on an orbital mixer. The sorbent was separated by centrifugation and uranium concentration measured by UV-visible spectroscopy using the Arsenazo (III) assay. Figure 4 shows the uranium sorption isotherms for the investigated mesoporous silica materials in water. Figure 5 shows the sorption isotherms in seawater.

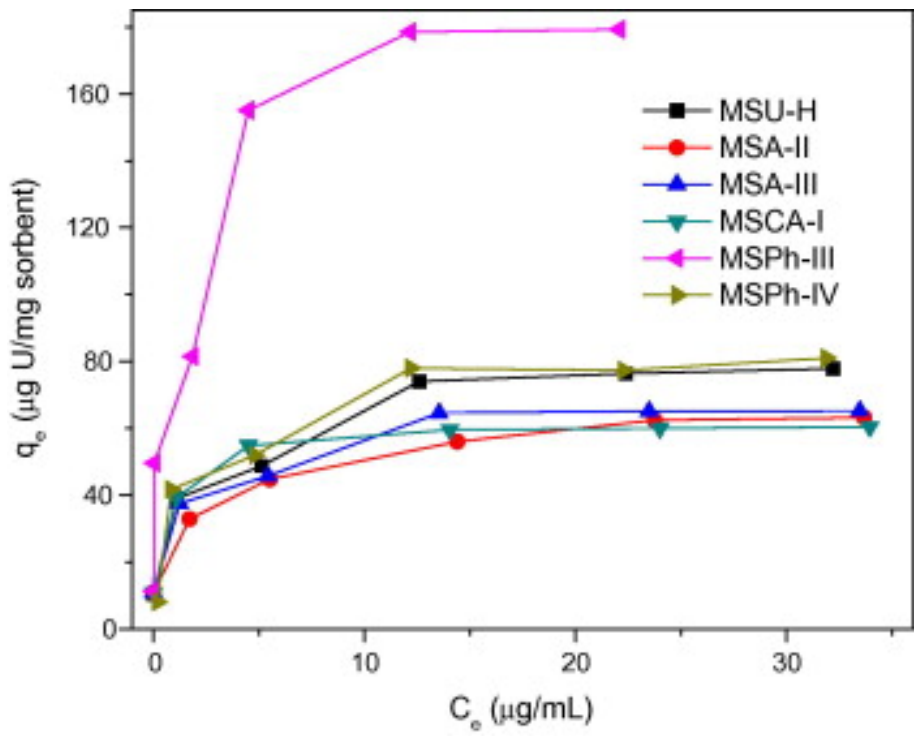

Figure 4. Uranium sorption isotherms for different mesoporous silica sorbents in water. $\mathrm{pH}=8.3+/-$ $0.1 ; m_{\text {sorbent }} / v_{\text {solution }}=0.1 \mathrm{mg} / \mathrm{mL}$. 
NEUP 11-3151

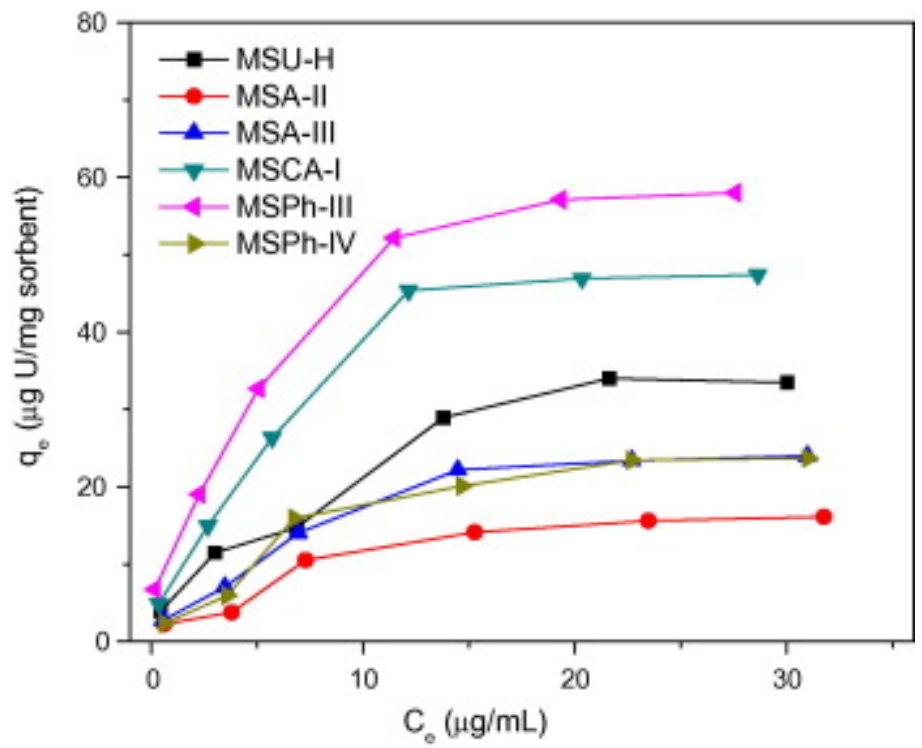

Figure 5. Uranium sorption isotherms for different mesoporous silica sorbents in seawater. $\mathrm{pH}=8.3+/$ $0.1 ; m_{\text {sorbent }} / v_{\text {solution }}=0.1 \mathrm{mg} / \mathrm{mL}$.

The Langmuir isotherm model assumes sorption occurs on an energetically homogeneous surface by monolayer deposition, with no interactions between adsorbates on adjacent sites. It can be expressed in its linear form as:

$$
\frac{C_{e}}{q_{e}}=\frac{C_{e}}{Q}+\frac{1}{Q \times b}
$$

where $C_{e}$ is the equilibrium concentration of uranium, $q_{e}$ is the amount of adsorbed metal at equilibrium (mg/g), and $Q$ and $b$ are Langmuir constants related to sorption capacity upon binding site saturation (mg/g) and affinity of the binding site on the sorbent $(\mathrm{mL} / \mathrm{mg})$, respectively. $Q$ and $b$ can be obtained by plotting $C_{e} / q_{e}$ versus $C_{e}$. Langmuir data for sorption in water and seawater are displayed in Tables 3 and 4 , respectively.

Table 3. Langmuir isotherm model parameters and correlation coefficients for uranium sorption by mesoporous silica sorbents in water

\begin{tabular}{|l|l|c|c|c|l|}
\hline Sample & $1 / Q$ & $1 /(Q \times b)$ & $\begin{array}{l}Q(\mu \mathrm{g} \mathrm{U} / \\
\text { mg sorbent })\end{array}$ & $b(\mathrm{~mL} / \mathrm{mg})$ & $R^{2}$ \\
\hline MSU-H & 0.012 & 0.017 & 80.6 & 0.74 & 0.993 \\
\hline MSA-II & 0.015 & 0.023 & 65.4 & 0.67 & 0.995 \\
\hline MSA-III & 0.015 & 0.015 & 67.6 & 1.00 & 0.996 \\
\hline MSCA-I & 0.016 & 0.006 & 61.0 & 2.78 & 0.999 \\
\hline MSPh-III & 0.005 & 0.004 & 185.2 & 1.32 & 0.991 \\
\hline MSPh-IV & 0.012 & 0.021 & 84.7 & 0.57 & 0.996 \\
\hline
\end{tabular}

Table 4. Langmuir isotherm model parameters and correlation coefficients for uranium sorption by mesoporous silica sorbents in seawater simulant

\begin{tabular}{|l|l|c|c|c|l|}
\hline Sample & $1 / Q$ & $1 /(Q \times b)$ & $\begin{array}{l}Q \text { ( } \mu \text { g U / } \\
\text { mg sorbent) }\end{array}$ & $b(\mathrm{~mL} / \mathrm{mg})$ & $R^{2}$ \\
\hline MSU-H & 0.023 & 0.18 & 43.1 & 0.13 & 0.933 \\
\hline
\end{tabular}




\begin{tabular}{|l|l|l|l|l|l|}
\hline MSA-II & 0.046 & 0.46 & 21.6 & 0.10 & 0.883 \\
\hline MSA-III & 0.032 & 0.26 & 31.1 & 0.12 & 0.965 \\
\hline MSCA-I & 0.017 & 0.10 & 58.1 & 0.18 & 0.975 \\
\hline MSPh-III & 0.015 & 0.06 & 66.7 & 0.26 & 0.979 \\
\hline MSPh-IV & 0.031 & 0.31 & 32.5 & 0.10 & 0.929 \\
\hline
\end{tabular}

In water the Langmuir model represents the adsorption of uranium particularly well, with correlation coefficients greater than 0.99 for each sorbent. Saturation capacity values for the amidoximefunctionalized materials were between $61.0 \mathrm{mg} / \mathrm{g}$ (MSCA-I) and $67.6 \mathrm{mg} / \mathrm{g}$ (MSA-III). Uranyl binding strength measurements revealed MSCA-I to have the strongest uranyl bonding, with a $b$ value of 2.78 $\mathrm{mL} / \mathrm{mg}$, which is consistent with the necessity of using $1 \mathrm{M} \mathrm{HCl}$ to achieve elution of the sorbed uranyl. MSA-II and MSA-III had values of 0.67 and 1.00, respectively. In seawater, the Langmuir isotherm represents the sorption data less well, with correlation coefficients between 0.883 and 0.975 . MSA-II and -III dropped in saturation capacity to less than half of the corresponding value obtained in water. Interestingly, MSCA-I decreased minimally, with a final value of $58.1 \mathrm{mg} / \mathrm{g}$. Binding strength decreased significantly for MSA-II and -III ( 0.10 and $0.12 \mathrm{mg} / \mathrm{mL}$, respectively) and by more than an order of magnitude for MSCA-I $(0.18 \mathrm{~mL} / \mathrm{mg})$. MSA-II and -III displayed S-type isotherms, suggesting more facile adsorption as concentration increases. This type of isotherm is common when there is strong competition for substrate sites, which may be due to $\mathrm{Ca}^{2+}$ and $\mathrm{Na}^{+}$in the seawater simulant. MSCA-I had a rare C-type isotherm, requiring a constant ratio of binding sites to sorbent despite increasing concentration and requiring more sites be made available as more solute is adsorbed. The BET isotherm shows a decrease in volume of gas adsorbed in the micropore region, indicative of sorbent grafting in both meso and micropores. Uranium may be rapidly transported through all pores and channels of the sorbent, binding initially to functional groups in the readily-accessible large pores, but also accessing the smaller pores as well. This could give the appearance of increased site availability and explain the C-type isotherm.

Sorption kinetics were obtained by sampling 10 different time points of sorbent in water and artificial seawater with a uranium concentration of $5 \mathrm{ppm}$. MS sorbents were added at a phase ratio of 0.1 $\mathrm{mg} / \mathrm{mL}$ and shaken at $300 \mathrm{rpm}$ on an orbital mixer throughout the duration of the time course. Sorbents were collected by centrifugation, and uranium concentration was measured by UV-visible spectroscopy using the Arsenazo (III) assay. Uranium sorption in MS materials is very rapid, with most uranium absorbed in the first 5-15 minutes, and equilibrium occurring in approximately 40 minutes. These results are consistent with the presence of large channels in the sorbent materials, and similar sorption kinetics have been reported previously. Modeling studies performed with homogeneously functionalized mesoporous silica reveal structural ordering is the dominant influence on accessibility to sorbent sites. Kinetic data for mesoporous silica materials in water and seawater simulant are displayed in Figures 6 and 7, respectively. 
NEUP 11-3151

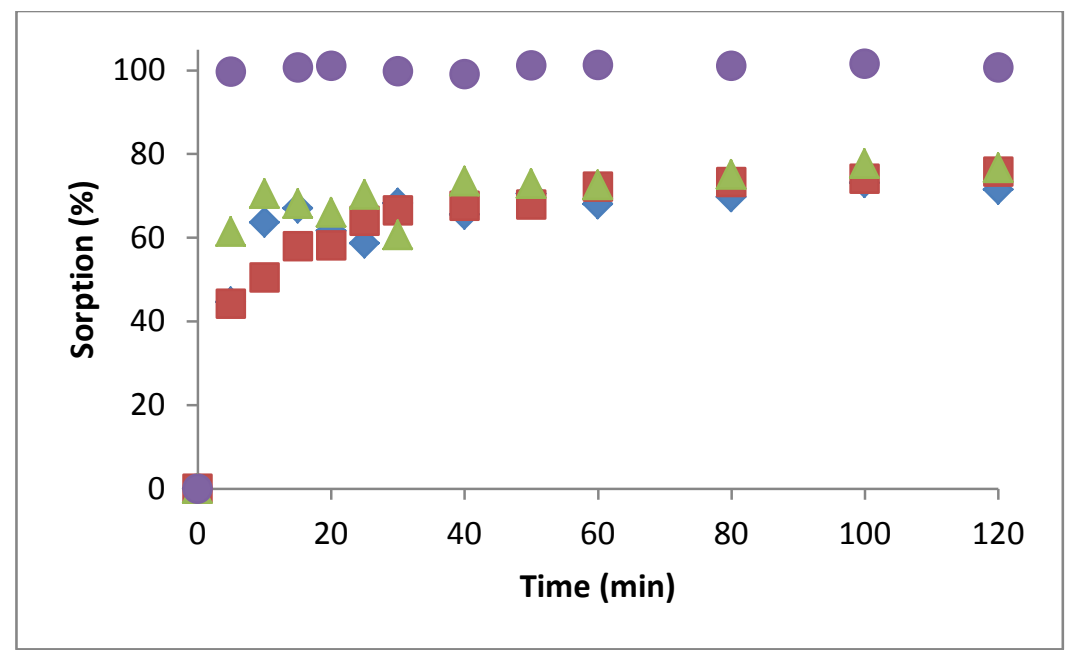

Figure 6. Kinetic studies on uranium sorption in MSA-II (diamonds), MSA-III (squares), MSCA-I (triangles), and MSPh-III (circles) in water. pH $8.3 \pm 0.1 ; m_{\text {sorbent }} / V_{\text {solution }}=0.1 \mathrm{mg} / \mathrm{mL}$; $[\mathrm{U}]_{\text {initial }}=$ 5 ppm.

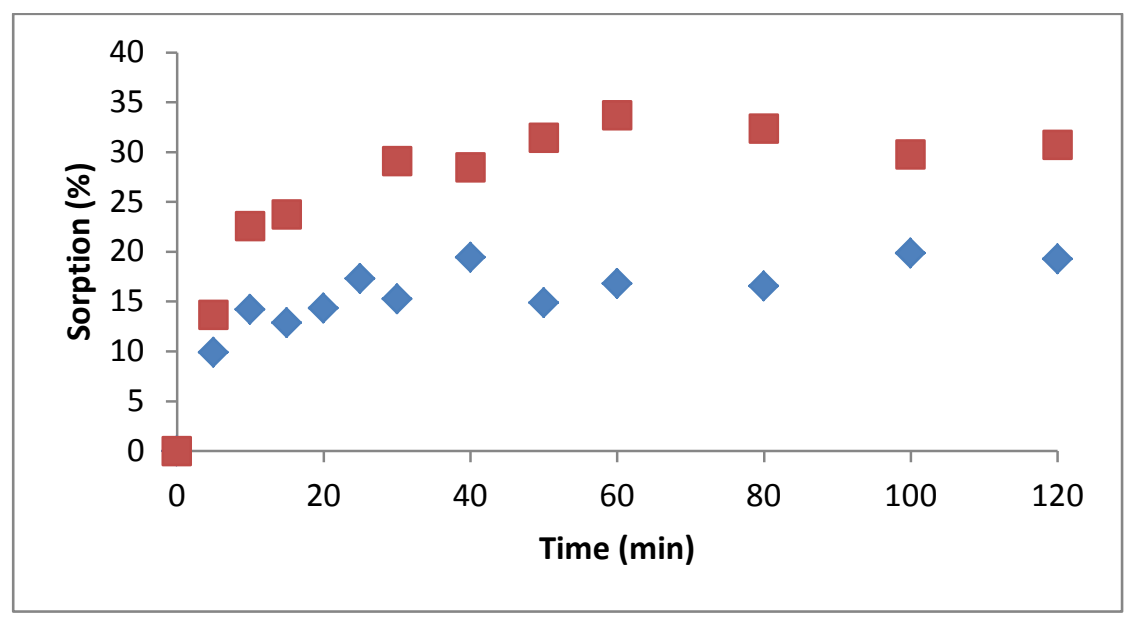

Figure 7. Kinetic studies on the U(VI) sorption in MSA-III (diamonds), and MSPh-III (squares) in artificial seawater. $\mathrm{pH} 8.3 \pm 0.1 ; m_{\text {sorbent }} / V_{\text {solution }}=0.1 \mathrm{mg} / \mathrm{mL} ;[\mathrm{U}]_{\text {initial }}=5 \mathrm{ppm}$.

Further optimization of MSN materials

Five additional non-amidoxime-based sorbents were investigated as grafted on MSU-H (Figure 8). One was a derivative of glycolic acid (MSGly), providing a carboxylic acid as the uranyl-binding moieity, while the other four were phosphonate-based sorbents (MSPh-I, -II, -III, and -IV). MSGly was previously grafted on a different mesoporous silica support and demonstrated to be an effective sorbent for actinide removal. By grafting it on MSU-H, this would provide a direct comparison for the binding group, eliminating any influence arising from the different support. Phosphorus-based materials such as tributyl phosphate (TBP) and carbamoylmethyphosphine oxide (CMPO) are known to complex strongly to uranium and other actinides, and are thus used in PUREX and TRUEX treatment processes. Furthermore, phosphonate-functionalized mesoporous silicas were recently demonstrated to be effective sorbents for removing uranium from aqueous matrices. The simple methyl phosphonate (MSPh-I) was designed to serve as a baseline material and to assess whether the absence of an oxygen would impede 
uranium sorption. The diethoxy-protected MSPh-II was previously studied and published, though again on a different support. The deprotected mono- and bis-functionalized analogs (MSPh-III and -IV) were prepared to investigate whether chelation of uranium might be facilitated by deprotonation of an acidic phosphoryl group.
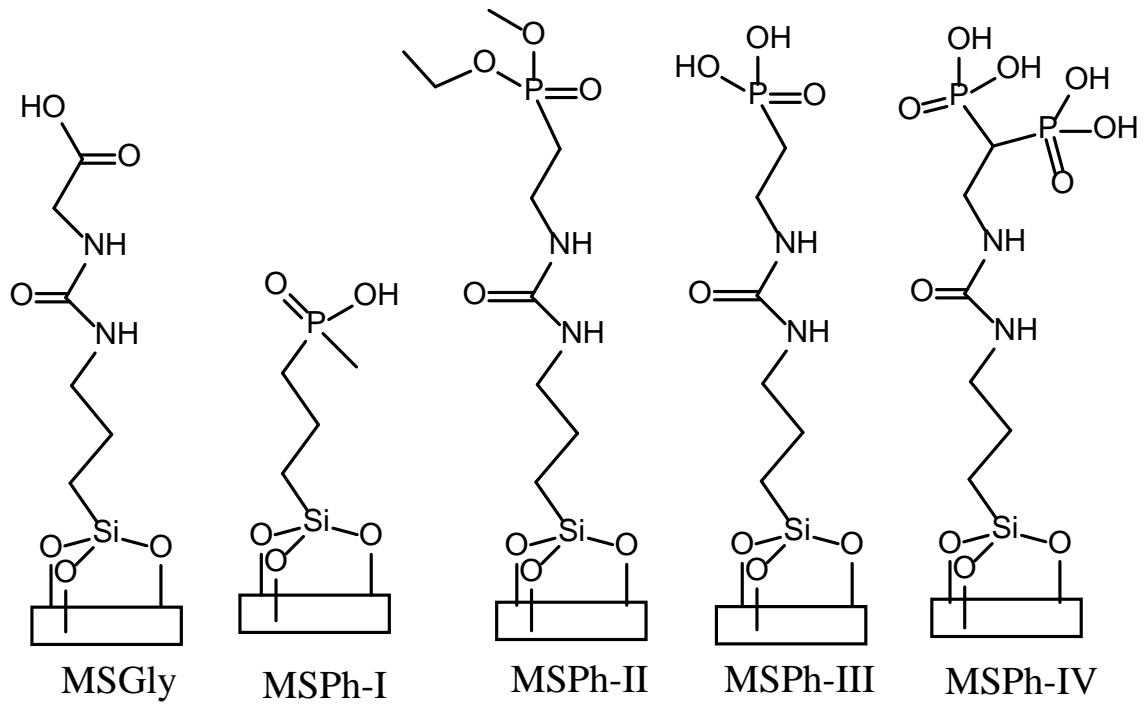

Figure 8. Phosphate- and carboxylate-derived organic ligands as grafted onto MSNs.

These materials were characterized in identical fashion to the amidoxime-functionalized materials. Thermogravimetric analysis indicate grafting densities of 0.82 to $1.38 \mathrm{mmol} / \mathrm{g}$ and BET surface areas range from 187 to $515 \mathrm{~m}^{2} / \mathrm{g}$, both of which are comparable to the materials prepared with amidoxime moieties and consistent with successful functionalization of the MSU-H support. IR spectra show a band at $1690-1650 \mathrm{~cm}^{-1}$ from stretching of the $\mathrm{C}=\mathrm{O}$ in the urea group. Finally, the $\zeta$-potential for these materials was shifted to more negative values of $-37.1,-20.7,-40.9,-46.6$, and $-44.6 \mathrm{mV}$ for $\mathrm{MSPh}-$ I, -II, -III, -IV, and MSGly, respectively. Characterization data for phosphate- and carboxylatefunctionalized MSNs are displayed in Table 5.

Table 5. Structural properties of phosphate- and carboxylate-derived mesoporous silica materials

\begin{tabular}{|l|c|c|c|c|}
\hline Material & $\begin{array}{l}\text { BET surface } \\
\text { area }\left(\mathrm{m}^{2} / \mathrm{g}\right)\end{array}$ & $\begin{array}{l}\text { BJH pore } \\
\text { size }(\mathrm{nm})\end{array}$ & $\begin{array}{l}\text { Loading } \\
(\mathrm{mmol} / \mathrm{g})\end{array}$ & $\zeta$-potential $(\mathrm{mV})$ \\
\hline MSPh-I & 515 & 3.8 & 1.38 & $-37.1 \pm 1.5$ \\
\hline MSPh-II & 227 & 6.6 & 1.02 & $-20.7 \pm 0.9$ \\
\hline MSPh-III & 406 & $7 / 8$ & 0.82 & $-40.9 \pm 1.5$ \\
\hline MSPh-IV & 253 & 5.6 & 1.02 & $-46.6 \pm 1.8$ \\
\hline MSGly & 187 & 4.9 & 1.25 & $-44.6 \pm 2.5$ \\
\hline
\end{tabular}

Preliminary uranyl sorption experiments performed in water revealed all materials removed between 40 to $50 \mathrm{mg}$ uranium/g sorbent, with no statistically significant difference between materials. These values are consistent with those of the amidoxime-functionalized materials, as displayed in Figure 2. In seawater, significant decreases in sorption were observed for all materials (Figure 3). While most phosphorous-based sorbents performed less well than the amidoxime-derived sorbents, MSPh-III 
possessed the highest uranium sorption capacity of all mesoporous silica materials, with $q_{e}$ of $13 \mathrm{mg} / \mathrm{g}$. Furthermore, elution studies revealed MSPh-III also requires washing with $1.0 \mathrm{M} \mathrm{HCl}$ to remove all sorbed uranium, similar to the strongly-binding MSCA-I. The following trend was observed for uranium absorption in seawater by mesoporous silica sorbents: MSPh-III > MSA-I MSA-II MSA-III MSCA-I > MSPH-II > MSGly MSPh-I MSPh-IV.

Isotherms were obtained for MSPh_III and MSPh-IV for comparison against the amidoximefunctionalized materials, discussed previously and shown in Figures 4 and 5. In water, the correlation coefficients for both phosphorus-based materials were greater than 0.99. MSPh-III had a saturation capacity of $185 \mathrm{mg} / \mathrm{g}$ and a uranyl binding strength of $1.32 \mathrm{~mL} / \mathrm{mg}$, second only to MSCA-I. MSPh-IV had a more modest saturation capacity of $85 \mathrm{mg} / \mathrm{g}$, and the lowest binding strength of $0.57 \mathrm{~mL} / \mathrm{mg}$. In seawater MSPh-III had the highest saturation capacity $(67 \mathrm{mg} / \mathrm{g})$ and best binding strength $(0.26 \mathrm{~mL} / \mathrm{mg})$, while MSPh-IV was similar to the amidoxime-functionalized materials in both saturation capacity (32 $\mathrm{mg} / \mathrm{g})$ and binding strength $(0.10 \mathrm{~mL} / \mathrm{mg})$. In seawater, MSPh-III exhibited an L-type isotherm, which occurs when there is competition for binding sites with increasing concentration, resulting in a gradual plateau to saturation. This is the traditional Langmuir isotherm, which occurs as a monolayer distribution is formed with no interaction between sorbents.

Studies with other nanoporous supports (MCNs, MOFs, COFs) including Uranyl sorption selectivity and kinetics studies

\section{Mesoporous Carbon Materials (MCNs):}

Despite efforts to minimize their effect, the presence of surface hydroxyl groups on the functionalized mesoporous silica supports obfuscate sorption data due to nonspecific physisorption interactions. Similar to mesoporous silica, mesoporous carbon nanoparticles (MCNs) exhibit high surface areas and large pore volumes. They are also robust under harsh conditions and show negligible background sorption of uranium prior to functionalization. To further investigate the efficacy of uranyl sorption, a series of ligands containing amidoxime, phosphoryl, and carboxyl groups were prepared and covalently grafted onto the surface of MCNs.

The library of ligands investigated included variations of the simple amidoxime and cyclic imide dioxime investigated with mesoporous silica. Six phosphonate or phosphoric acid derived ligands were investigated, as were four carboxylate ligands. Additionally, two materials were prepared by functionalizing first with amidoxime or cyclic imide dioxime, followed by subsequent functionalization with a carboxylic acid group. It was hoped cooperative interactions between these binding groups might facilitate higher uranium uptake. The ligands used for functionalizing MCNs are shown in Figure 9.

Most sorbent groups were synthesized as substituents on the para position of aniline, allowing for straightforward grafting onto the MCN through in situ formation of an aryl diazonium cation and subsequent covalent bond formation. Alternative methods of functionalization were also explored, including simple oxidation of the starting material with $\mathrm{HNO}_{3}$ to graft carboxyl groups directly onto the MCN, as well as phosphorylation with $\mathrm{H}_{3} \mathrm{PO}_{4}$. The bisphosphonate ligand, MC-bis( $\left.\mathrm{PO}(\mathrm{OH})_{2}\right)$, was prepared through formation of an acid chloride, followed by nucleophilic substitution by alendronate. 


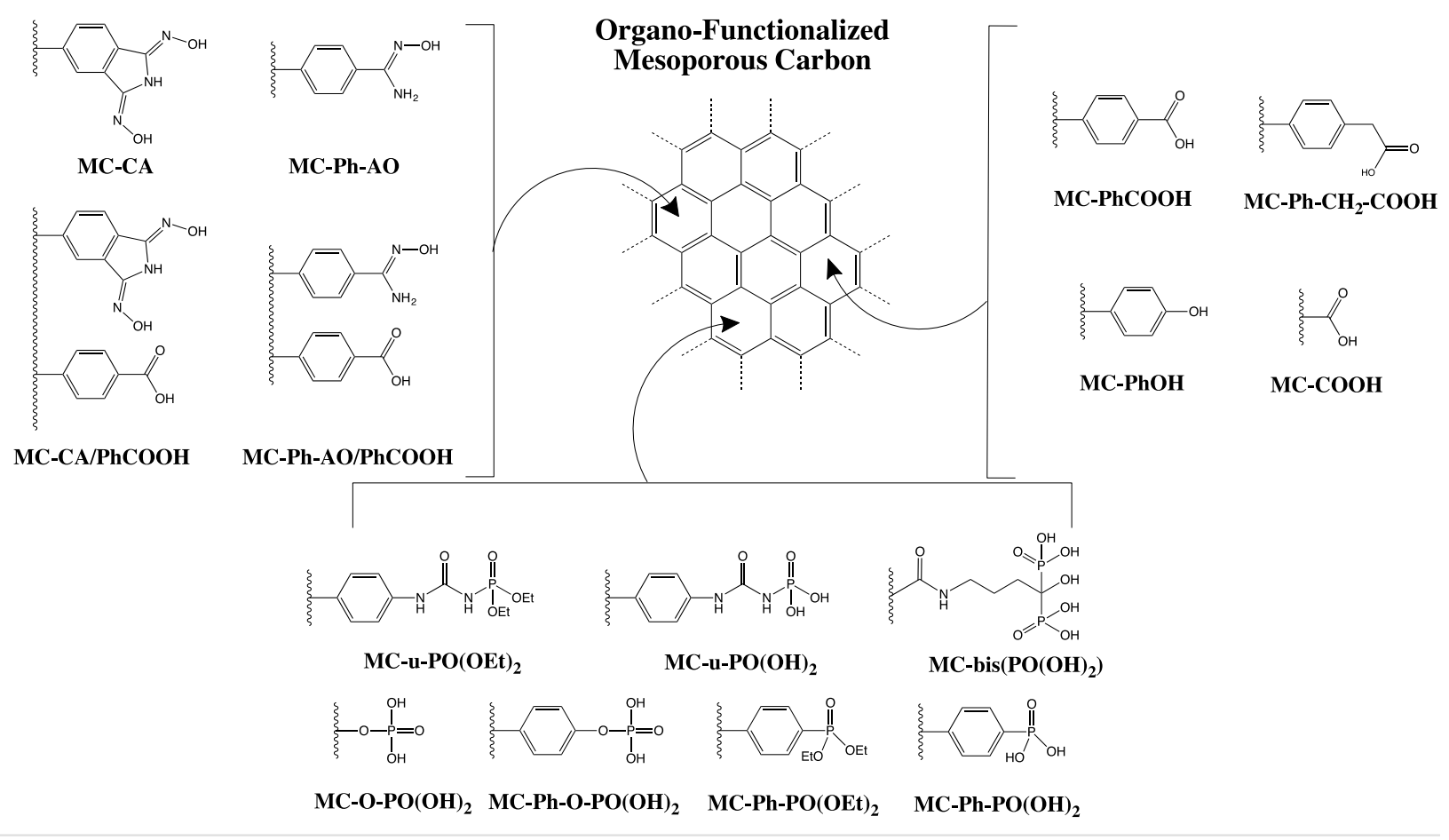

Figure 9. Organo-functionalized mesoporous carbon nanomaterial (MCN) sorbents investigated for uranium sorption.

All materials were characterized by thermogravimetric analysis and BET surface area and investigated for uranyl sorption. Characterization data is summarized in Table 6. Nitrogen sorption isotherms exhibit features consistent with functionalized mesoporous materials. Specific surface areas were obtained by applying the BET method, while average pore size was determined by the BarrettJoyner-Halenda (BJH) method. Surface areas on the functionalized materials ranged from 989 to 163 $\mathrm{m}^{2} / \mathrm{g}$, with average pore diameters showing a broad distribution around $8 \mathrm{~nm}$. The surface areas of the organo-functionalized MC materials were smaller than that of the starting material. This decrease in surface area is consistent with functionalization of the materials and the preservation of readily accessible pores, as was previously observed with mesoporous silica.

TGA analysis of the unfunctionalized MCN material showed no significant weight loss prior to the distinct onset of material decomposition around $700{ }^{\circ} \mathrm{C}$. In contrast, organo-functionalized MCNs showed weight loss between $300-700{ }^{\circ} \mathrm{C}$, indicating functional groups were covalently attached to the surface of the carbon material, and also facilitating calculation of the functional group loadings. Based on these data, the MCNs had grafting densities ranging from 0.13 to1.82 mmol/g, which are largely comparable with the grafting densities of functionalized mesoporous silica.

Table 6. Porosities and functional group loadings of organo-functionalized mesoporous carbon nanomaterials

\begin{tabular}{|l|c|c|}
\hline Materials & $\begin{array}{l}\text { BET surface } \\
\text { area }\left(\mathrm{m}^{2} / \mathrm{g}\right)\end{array}$ & $\begin{array}{l}\text { Loading } \\
(\mathrm{mmol} / \mathrm{g})\end{array}$ \\
\hline MC (unfunctionalized) & 189 & --- \\
\hline MC-CA & 148 & 1.82 \\
\hline
\end{tabular}




\begin{tabular}{|l|c|c|}
\hline MC-Ph-AO & 109 & 0.83 \\
\hline MC-CA/PhCOOH & 125 & 0.52 \\
\hline MC-Ph-AO/PhCOOH & 138 & 0.62 \\
\hline MC-Ph-COOH & 163 & 0.45 \\
\hline MC-Ph-CH ${ }_{2}-\mathrm{COOH}$ & 98 & 1.17 \\
\hline MC-COOH & 174 & 0.83 \\
\hline MC-Ph-OH & 138 & 1.52 \\
\hline MC-Ph-O-PO(OH $)_{2}$ & 157 & 1.05 \\
\hline MC-O-PO(OH $)_{2}$ & 154 & 0.28 \\
\hline MC-bis(PO(OH $\left.)_{2}\right)$ & 122 & 0.29 \\
\hline MC-u-PO(OEt $)_{2}$ & 93 & 1.11 \\
\hline MC-u-PO(OH $)_{2}$ & 105 & 0.50 \\
\hline MC-Ph-PO(OEt $)_{2}$ & 174 & 0.13 \\
\hline MC-Ph-PO(OH $)_{2}$ & 179 & 0.16 \\
\hline
\end{tabular}

Functionalized MCNs were investigated for uranium extraction under conditions intended to simulate seawater and acid mine drainage, plotted in Figure 10 and summarized in Table 7. Acid mine drainage simulant had a $\mathrm{pH}$ of 4 and uranium concentration of $100 \mathrm{ppm}$. In contrast, simulated seawater had a $\mathrm{pH}$ of 8.2 and a uranium concentration of $5 \mathrm{ppm}$. Under acidic conditions, sorption ranged from 64 to $1.6 \mathrm{mg}$ of uranium per gram of sorbent, depending upon the functional ligand grafted to the material. Normalizing to account for the grafting density did not significantly change the relative sorption capacity of the materials investigated. The cyclic imide dioxime exhibited a higher sorption capacity (13 mg/g) than materials functionalized with simple amidoxime $(6 \mathrm{mg} / \mathrm{g})$. Co-grafting with carboxyl groups significantly increased the sorption of both materials to 18 and $22 \mathrm{mg} / \mathrm{g}$ for co-grafted cyclic imide dioxime and amidoxime, respectively. While these results are statistically equivalent to the sorption observed for simple functionalization with a carboxylic acid, the observed loading indicates more uranium was bound per sorbent group than for either functional group grafted in isolation. It is possible this could be attributed to cooperative binding interactions, and further investigations are ongoing to more extensively probe this phenomenon.

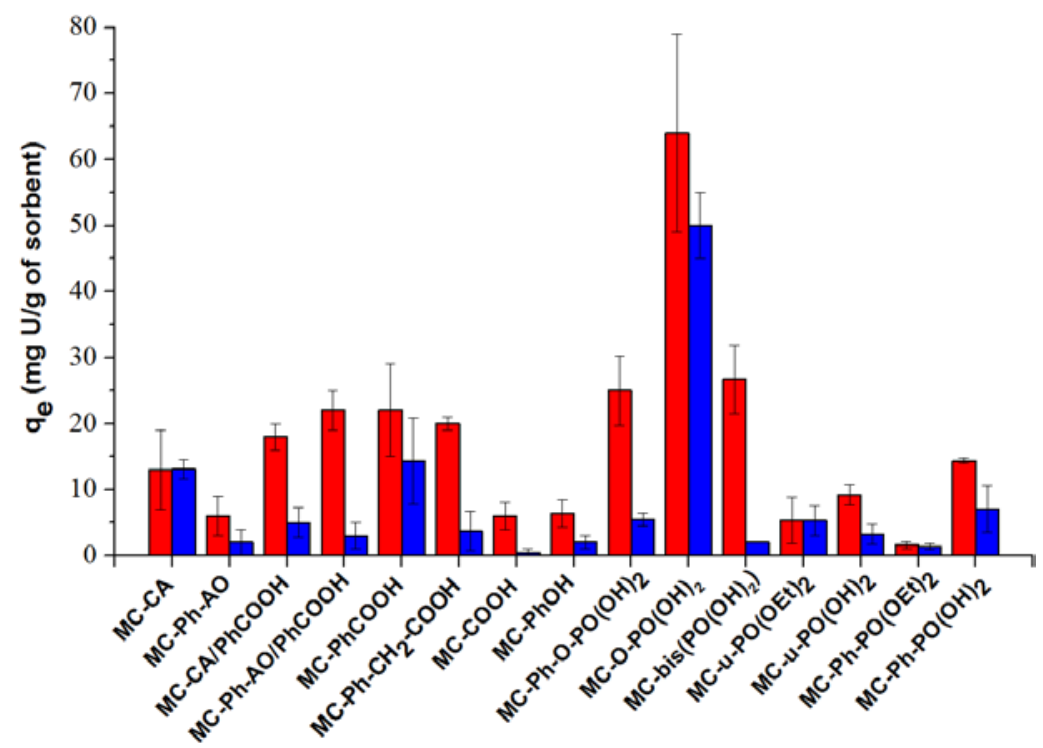

Figure 10. Uranium sorption capacities of $\mathrm{MCN}$ materials in acidic water at $\mathrm{pH}=4$ (red) and in artificial seawater at $\mathrm{pH}=8.2$ (blue). 
NEUP 11-3151

Table 7. Uranium sorption capacities of MCN materials in acidic water at $\mathrm{pH}=4$ and in artificial seawater at $\mathrm{pH}=8.2$.

\begin{tabular}{|l|c|c|}
\hline Materials & Water $(\mathrm{mg} / \mathrm{g})$ & Seawater $(\mathrm{mg} / \mathrm{g})$ \\
\hline MC (unfunctionalized) & --- & --- \\
\hline MC-CA & $13.0 \pm 6.0$ & $13.1 \pm 1.5$ \\
\hline MC-Ph-AO & $6.0 \pm 3.0$ & $2.0 \pm 2.0$ \\
\hline MC-CA/PhCOOH & $18.0 \pm 2.0$ & $5.0 \pm 2.2$ \\
\hline MC-Ph-AO/PhCOOH & $22.0 \pm 3.0$ & $3.0 \pm 2.0$ \\
\hline MC-Ph-COOH & $22.0 \pm 3.0$ & $14.3 \pm 6.5$ \\
\hline MC-Ph-CH ${ }_{2}-\mathrm{COOH}$ & $20.0 \pm 1.0$ & $3.7 \pm 3.0$ \\
\hline MC-COOH & $6.0 \pm 2.0$ & $0.5 \pm 0.5$ \\
\hline MC-Ph-OH & $6.4 \pm 2.0$ & $2.0 \pm 1.0$ \\
\hline MC-Ph-O-PO(OH $)_{2}$ & $25.0 \pm 5.3$ & $5.5 \pm 1.0$ \\
\hline MC-O-PO $(\mathrm{OH})_{2}$ & $64.0 \pm 15.0$ & $50.0 \pm 5.0$ \\
\hline MC-bis $\left(\mathrm{PO}(\mathrm{OH})_{2}\right)$ & $26.7 \pm 5.2$ & $2.0 \pm 0.0$ \\
\hline MC-u-PO(OEt $)_{2}$ & $5.3 \pm 3.5$ & $0.3 \pm 2.2$ \\
\hline MC-u-PO(OH $)_{2}$ & $9.2 \pm 1.5$ & $3.2 \pm 1.5$ \\
\hline MC-Ph-PO $(\mathrm{OEt})_{2}$ & $1.6 \pm 0.5$ & $1.4 \pm 0.5$ \\
\hline MC-Ph-PO(OH $)_{2}$ & $14.4 \pm 0.4$ & $7.0 \pm 3.5$ \\
\hline
\end{tabular}

Simple phosphorylation of the MCN (MC-O-PO(OH $)_{2}$ ) resulted in the highest sorption capacity of the MCN functionalized materials (64 mg/g). This result is particularly remarkable as the material also had one of the lowest loadings. Efforts to increase sorption by separating the phosphoric acid functional group from the walls of the MCNs were unsuccessful, as each attempt resulted in lower overall sorption. Diethoxy- and dihydroxyphosphorylurea-grafted MCNs were also prepared, though relatively low sorption capacities were obtained. The bisphosphonyl-functionalized material (MC-bis $\left(\mathrm{PO}(\mathrm{OH})_{2}\right)$, had a sorption capacity comparable to the monophosphonyl-functionalized material, despite containing two uranium binding sites.

In artificial seawater, uraniuim sorption decreased for all materials, presumably due to the formation of the highly stable uranium carbonate complexes that predominate at higher $\mathrm{pH}$. Competition from other ions in seawater is also known to decrease uranium binding. Similar to the studies under acidic conditions, the phosphorylated MCN MC-O-PO(OH $)_{2}$ exhibited the largest sorption capacity of 50 $\mathrm{mg} / \mathrm{g}$. Under the experimental conditions, this constitutes complete sorption of all uranium in solution. Sorption capacities for some of the other sorbents were dramatically affected by this change in medium. Most notably, significant decreases were observed for two of the better-performing sorbents under acidic conditions, MC-bis $\left(\mathrm{PO}(\mathrm{OH})_{2}\right)$ and $\mathrm{MC}-\mathrm{Ph}-\mathrm{O}-\mathrm{PO}(\mathrm{OH})_{2}$. Equally interesting, the cyclic imine dioxime (MC-CA) and MC-u-PO $(\mathrm{OEt})_{2}$ did not change significantly upon switching medium. Both of these ligands can coordinate uranium in a multidentate fashion and could thus exhibit some selectivity which may be worth further investigation. 
NEUP 11-3151

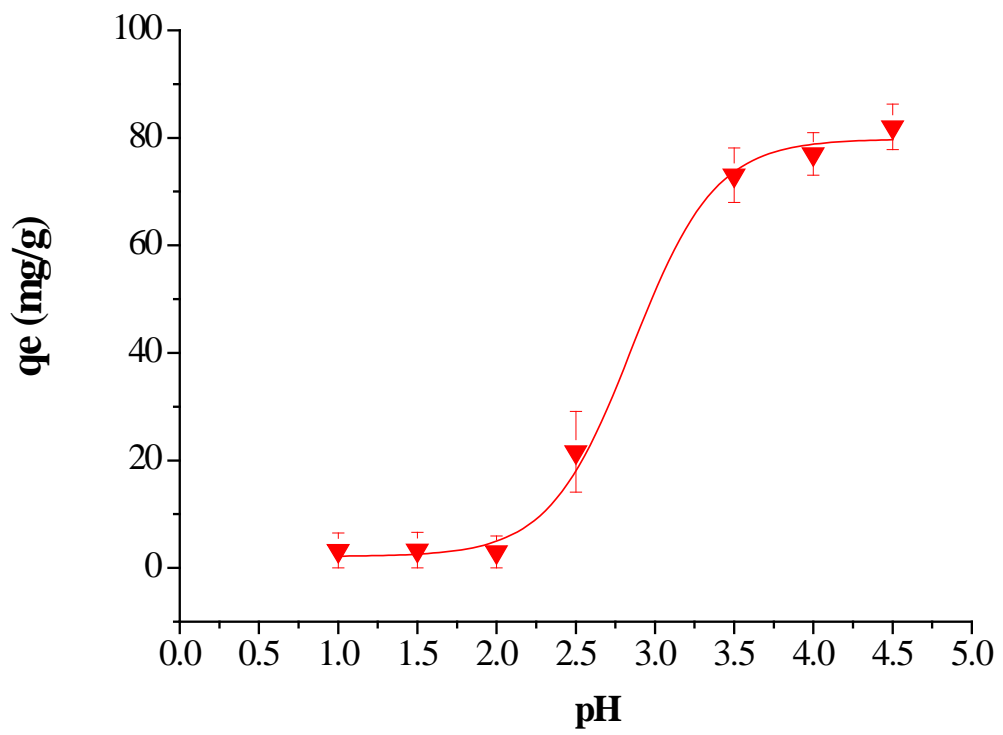

Figure 11. Effect of $\mathrm{pH}$ on adsorption of uranium by $\mathrm{MC}-\mathrm{O}-\mathrm{PO}(\mathrm{OH})_{2} . \mathrm{V}=10 \mathrm{~mL}, \mathrm{~m}=10 \mathrm{mg}$, [U] = 100 ppm.

Detailed sorption studies were performed using the material which exhibited the greatest uranium sorption capacity, $\mathrm{MC}-\mathrm{O}-\mathrm{PO}(\mathrm{OH})_{2}$. Sorption with respect to loading revealed that $96 \%$ and $75 \%$ of phosphoryl moieties were coordinated by uranium in acidic water and seawater simulant, respectively. Subsequent experiments revealed sorption of uranium is heavily influenced by the $\mathrm{pH}$ of solution due to the effects of $\mathrm{pH}$ on the solubility of the uranyl cation, speciation of uranium, and the charge on the functional group. A batch assay for uranyl sorption with $\mathrm{MC}-\mathrm{O}-\mathrm{PO}(\mathrm{OH})_{2}$ was made of the $\mathrm{pH}$ range from 1 to 4.5 using a $100 \mathrm{ppm}$ uranium solution in water with a one hour contact time. The results reveal that uranium uptake with this material increased abruptly from $\mathrm{pH} 2.5$ to approximately $\mathrm{pH} 3.5$, after which it plateaued (Figure 11). The dramatic increase can be attributed to the first deprotonation of the phosphoryl groups, which can be approximated with the $\mathrm{pK}_{\mathrm{a} 1}$ of phosphoric acid, 2.12. Deprotonation of the organic group resulted in an approximate 7-fold increase in sorption between $\mathrm{pH} 1.5$ and $\mathrm{pH} 4$. Under these conditions, uranyl is known to form a 2+ charged penta hydrate complex with all coordination filled with labile aqua ligands. It can be rationalized that the increase in sorption is due to the ionic interaction between a cationic uranyl molecule and an anionic dihydrogen phosphate moiety. Extrapolation to other functionalized MCN materials could provide insight into the observed sorption. Sorbents expected to bind uranium through ionic interactions would see an increase in sorption at $\mathrm{pH}$ values above their $\mathrm{pK}_{\mathrm{a}} \mathrm{s}$, with resonance stabilization of the anionic species being an important characteristic of a good sorbent. Suppression of sorption by such interactions could be expected in seawater because of competing ions and the formation of the tetranionic uranyl carbonate.

Sorption isotherms were obtained by varying the uranium concentration in solution and measuring the subsequent sorption. We observed an increase in uranium extraction with increasing uranium concentration, ultimately reaching a plateau upon saturation of sorbent groups. Data were evaluated using the Langmuir model, described above. This model assumes monolayer sorption on an energetically homogeneous surface by monolayer deposition with no interaction between adsorbates on adjacent sites. The equilibrium data fit the Langmuir model well, with saturation capacities of $97 \mathrm{mg} / \mathrm{g}$ and $67 \mathrm{mg} / \mathrm{g}$ obtained in acidic water and seawater simulant, respectively. Sorption isotherms in acidic water and simulated seawater are displayed in Figure 12 with Lanmuir model parameters in Table 8. 

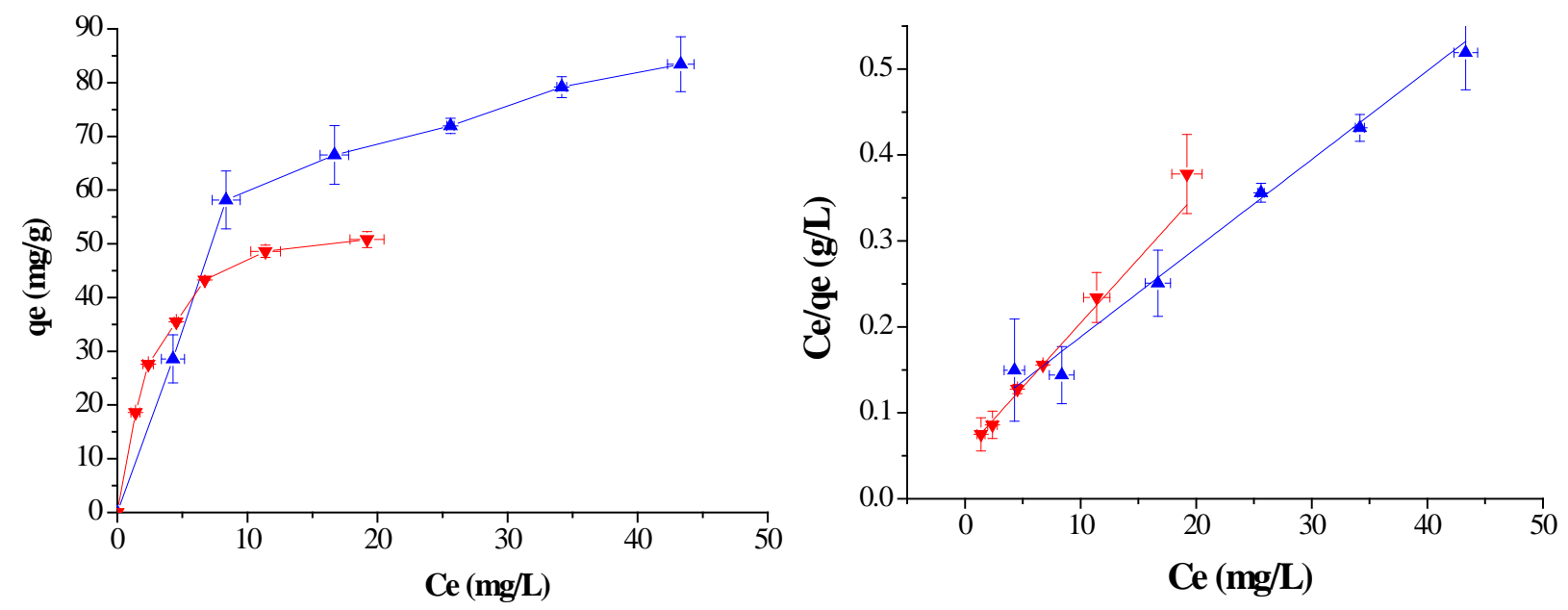

Figure 12. (Left) Sorption isotherm of $\mathrm{MC}-\mathrm{O}-\mathrm{PO}(\mathrm{OH})_{2}$ at $\mathrm{pH}=4$ in water (red) and in artificial seawater at $\mathrm{pH}=8.2$ (blue). Error bars are the standard deviation of each point, with experiments performed in triplicate. (Right) Sorption isotherms displayed in their linearized format and fit by the Langmuir model. Values for saturation sorption capacity $\left(q_{\max }\right)$, and the Langmuir constant $\left(K_{L}\right)$ can be obtained from the slope and y-intercept.

Table 8. Langmuir model parameters for $\mathrm{U}$ sorption onto $\mathrm{MC}-\mathrm{O}-\mathrm{PO}(\mathrm{OH})_{2}$ in acidic water $(\mathrm{pH}=4)$ and artificial seawater $(\mathrm{pH}=8.2)$

\begin{tabular}{|l|c|c|c|c|c|}
\hline & $1 / q_{\max }$ & $1 / K_{L} q_{\max }$ & $q_{\max }(\mu \mathrm{g} / \mathrm{mg})$ & $K_{L}(\mathrm{~mL} / \mathrm{mg})$ & $\mathbf{R}^{2}$ \\
\hline Acidic Water & $0.01033 \pm$ & $0.08495 \pm$ & $96.8 \pm 8.9$ & $0.1216 \pm$ & 0.981 \\
& 0.00105 & 0.02935 & & 0.0388 & \\
\hline Seawater & $0.01492 \pm$ & $0.05532 \pm$ & $67.1 \pm 6.5$ & $0.269 \pm$ & 0.978 \\
Simulant & 0.00157 & 0.01046 & & 0.065 & \\
\hline
\end{tabular}

Data were also modeled by the Freundlich and Temkin isotherms. The Freundlich model is commonly used to describe sorption on heterogeneous surfaces, and the Temking model is used to take adsorbent-adsorbate interactions into account. These models are expressed as

$$
\begin{gathered}
\ln \left(q_{e}\right)=\ln (k)+\frac{1}{n} \ln \left(C_{e}\right) \\
q_{e}=B \ln \left(A_{T}\right)+B \ln \left(C_{e}\right)\left(B=\frac{R T}{b_{T}}\right)
\end{gathered}
$$

where $k(\mu \mathrm{g} / \mathrm{g})$ and $n$ are the Freundlich constants related to sorption capacity and sorption intensity, respectively, and $B, A_{T}$, and $b_{T}$ are the Temking constants related to the heat of sorption, the equilibrium binding constant, and the Temkin constant. $R$ is the universal gas constant $[8.314 \mathrm{~J} /(\mathrm{mol} \mathrm{K})]$, and $T$ is the temperature. These parameters confirm a better affinity for uranium sorption by the material in acidic water than in seawater.

Sorption kinetics of uranium in $\mathrm{MC}-\mathrm{O}-\mathrm{PO}(\mathrm{OH})_{2}$ were studied in both water and artificial seawater with solutions of 200 and 100 ppm uranium, respectively Under both types of conditions, uranium sorption with $\mathrm{MC}-\mathrm{O}-\mathrm{PO}(\mathrm{OH})_{2}$ can be separated into two steps: a first step in which the majority 
of uranium is adsorbed in less than five minutes, and a slower second step, ultimately reaching equilibrium. Plots of kinetic time course measurements are displayed in Figure 13.
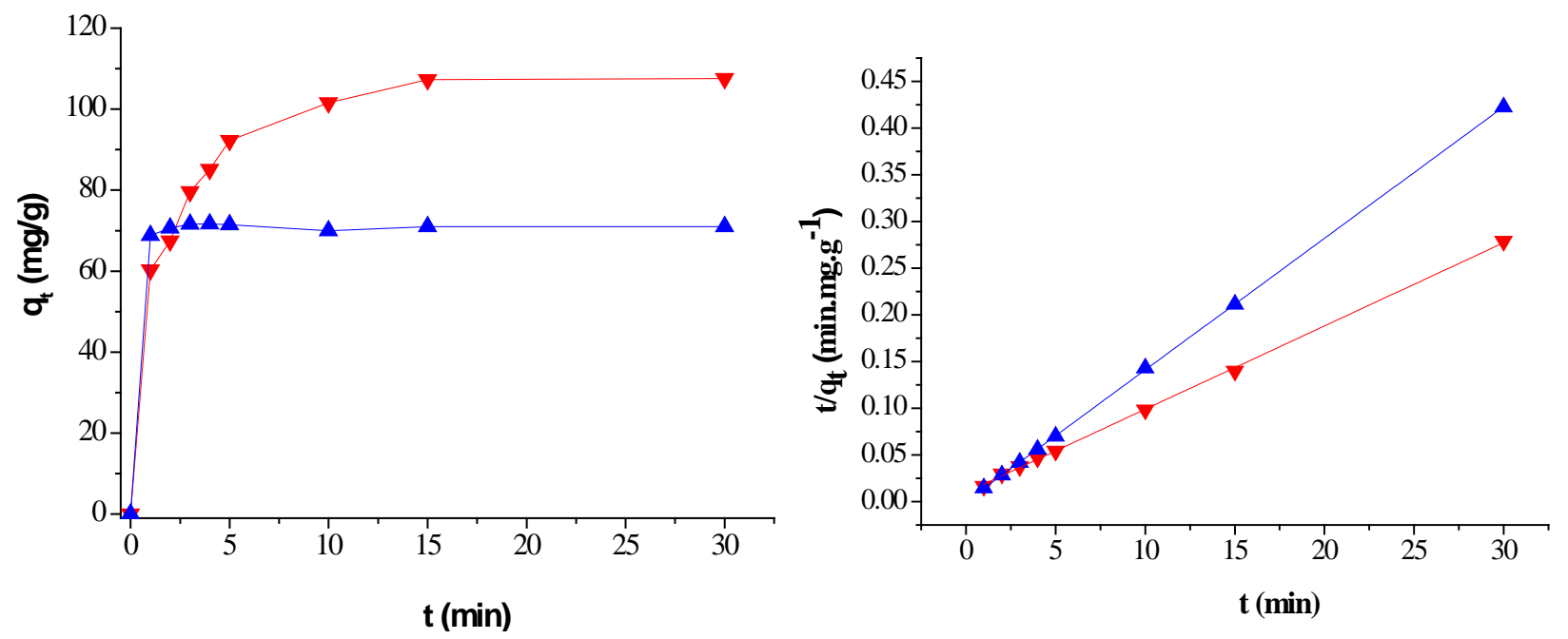

Figure 13. (Left) Kinetic studies of $U$ sorption by $\mathrm{MC}-\mathrm{O}-\mathrm{PO}(\mathrm{OH})_{2}$ in $200 \mathrm{ppm} \mathrm{U}$ acidic water (red) and 100 ppm U artificial seawater (blue) during the first 30 minutes. No additional sorption is observed after this time. (Right) Pseudo second-order plot of $\mathrm{U}$ sorption by $\mathrm{MC}-\mathrm{O}-\mathrm{PO}(\mathrm{OH})_{2}$ in acidic water (red) and artificial seawater (blue).

To evaluate the diffusion mechanism of uranium inside the material and the rate-determining step, the data were fit by the intraparticle diffusion model, expressed as

$$
q_{t}=k_{i d} t^{\frac{1}{2}}
$$

where $k_{i d}$ is the intraparticle diffusion rate constant. If the rate of uranium sorption is limited by intraparticle diffusion, a plot of $q_{t}$ as a function of $t^{1 / 2}$ must be linear and pass through the origin. The noncorrelation of the data with these requirements indicate that intraparticle diffusion is not ratedetermining throughout the entire sorption process, and that different mechanisms are involved in uranium uptake. Intraparticle diffusion rate constants were obtained from the slopes of the linear portions of the respective plots. The initial uptake of uranium can be attributed to sorption at readily available binding sites on or near the surface of the material, whereas the second step results from diffusion of the uranium ions through the pores of the material to be bound by interior sorbent groups.

The very rapid sorption and abrupt plateau in seawater simulant might be the result of competing ions more rapidly blocking inner sorbent sites, as their smaller atomic radii facilitate more rapid mass transport than for uranyl carbonate. As a result, the majority of uranium sorption occurs rapidly and on the surface of the material. In contrast, because of the absence of any competing ions under acidic conditions, uranium is capable of gradually diffusing through the pores of the MC material. The slower saturation kinetics and higher sorption capacity are readily explained by this rationale.

The adsorption mechanism was also investigated by fitting the experimental data to pseudo-firstand pseudo-second-order rate equations. The linear form of the pseudo-first-order rate equation is expressed as:

$$
\ln \left(q_{e}-q_{t}\right)=\ln \left(q_{e}\right)-k_{1} t
$$

The linear form of the pseudo-second-order rate equation is expressed as: 


$$
\frac{t}{q_{t}}=\frac{1}{k_{2} q_{e}^{2}}+\frac{t}{q_{e}}
$$

For both equations, $q_{e}$ and $q_{t}$ are the sorption capacities at equilibrium at time $t$, respectively, and $k_{1}$ and $k_{2}$ are the pseudo-first- and pseudo-second-order rate constants, respectively. The pseudo-first-order kinetic model was initially applied. Under acidic conditions, this model fit the data well, yielding $k_{1}=0.35 \mathrm{~min}^{-1}$ with $R^{2}=0.948$. However, this model did not accurately fit the data obtained under seawater conditions. Application of the pseudo-second-order model yielded very good linearity, with correlation coefficients of 0.99936 under acidic conditions and 0.99996 in artificial seawater. This model was then applied to predict the rate constants and equilibrium capacities under both types of conditions. The saturation capacities obtained from the isotherms and kinetic analyses are in good agreement, as displayed in Table 9.

Table 9. Saturation capacities obtained for mesoporous carbon nanomaterials by uranium sorption isotherms and kinetic analyses in acidic water and seawater simulant.

\begin{tabular}{|c|c|c|}
\hline Parameters & Acidic Water & Artificial Seawater \\
\hline$q_{e}($ exp. $)(\mathrm{mg} / \mathrm{g})$ & $96.8 \pm 8.9$ & $67.1 \pm 6.5$ \\
\hline$k_{2}(\mathrm{~g} / \mathrm{mg} / \mathrm{min})$ & $0.00816 \pm 0.00015$ & $1.858 \pm 1.48$ \\
\hline$q_{e}($ calc. $)(\mathrm{mg} / \mathrm{g})$ & $112.1 \pm 1.1$ & $70.9 \pm 0.2$ \\
\hline $\mathbf{R}^{2}$ & 0.99936 & 0.99996 \\
\hline
\end{tabular}

\section{Metal-organic Frameworks:}

Metal-organic frameworks (MOFs) are a class of hybrid materials composed of organic bridging ligands coordinated to metal ions or metal ion clusters. Possessing high porosities and ready tunability, these materials have shown promise for diverse applications including catalysis, sensing, nonlinear optics, separations, drug delivery, and gas storage. MOFs have also been used to sorb and contain volatile radionuclides, as ZIF-8 was used to absorb molecular $\mathrm{I}_{2}$ under atmospheric pressures. Irreversible containment was achieved through pressure-induced amorphization, and subsequent sintering rendered a monolithic material suitable for long-term storage.

MOFs must possess adequate stability in aqueous solution for potential application in uranium extraction. In addition, they must have large pore apertures to facilitate the transport of hydrated actinide ions through the interior of the frameworks. UiO MOFs possess high stability in aqueous solution due to robust $\mathrm{Zr}_{6} \mathrm{O}_{4}(\mathrm{OH})_{4}$ (carboxylate) $)_{12}$ secondary building units (SBUs), while their open channels are easily tuned by varying the lengths of the dicarboxylate bridging ligand. Even with the amino-TPDC $\left(\mathbf{L}_{1}\right)$ as the bridging ligand, the UiO-68- $\mathrm{NH}_{2}$ framework (MOF 1) has large tetrahedral pores with apertures that can accommodate particles of up to $10 \AA$ in diameter. To investigate their potential as uranium sorbents, we modified the amine group of amino-TPDC to phosphorylurea for the synthesis of UiO MOFs as novel sorbent materials with precisely known loadings of actinide-binding moieties.

The $N$-diphenylphosphorylurea (DPPU) functional group was previously shown to extract actinides and lanthanides from nitric acid solutions. Similar in structure to the well-known carbamoylphosphine oxides (CMPOs) used in the TRUEX process, phosphorylureas have a secondary amine between phosphonyl and carbonyl groups as opposed to a methylene group in CMPOs. A recent computational study suggests this replacement ensures delocalization of the electron system of DPPU and enhances efficiency in cation binding. We anticipated the preparation of a promising sorbent material for actinides by combining the high porosity and stability of UiO MOFs with the phosphorylurea ligand's affinity for actinides. 


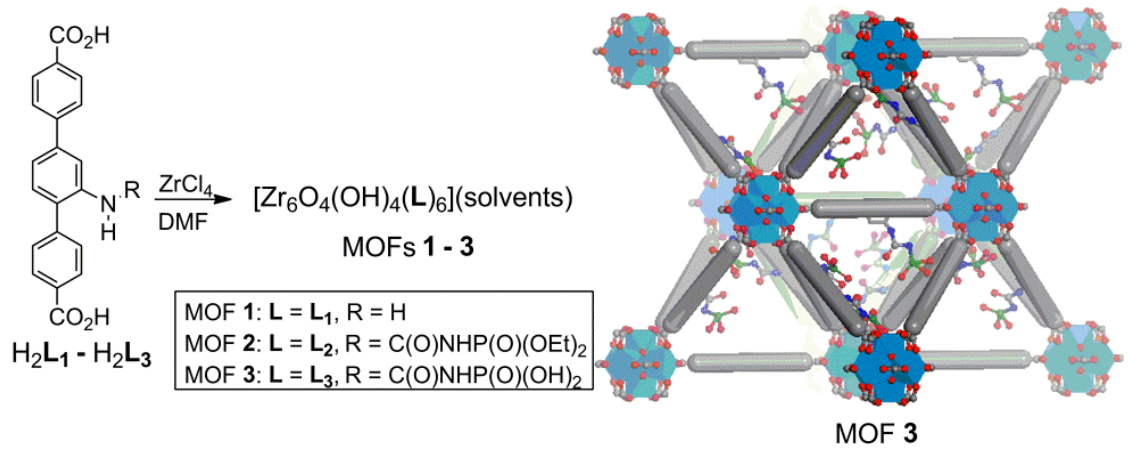

Figure 14. Schematic showing the preparation of MOFs 1-3 and a structure model of the new UiO MOF (3) displaying the readily accessible phosphorylurea groups.

Diethoxyphosphorylurea-derived TPDC ligand $\left(\mathrm{H}_{2} \mathbf{L}_{2}\right)$ was prepared in quantitative yield by condensation of the amino- $\mathrm{H}_{2}$ TPBC with commercially available diethoxyphosphinyl isocyanate. The new UiO MOF containing the diethoxyphosphorylurea group [UiO-68-P(O)(OEt) $)_{2}$ MOF 2] was prepared by heating a solution of $\mathrm{ZrCl}_{4}, \mathrm{H}_{2} \mathbf{L}_{2}$, and benzoic acid in DMF at $70{ }^{\circ} \mathrm{C}$ for 3 days (Figure 14). The resulting light yellow powder was collected by centrifugation and washed with DMF and ethanol. Subsequent deprotection of 2 with $\mathrm{Me}_{3} \mathrm{SiBr}$ followed by hydrolysis with water yielded the isomorphous MOF functionalized with dihydroxyphosphorylurea groups [UiO-68- $\mathrm{P}(\mathrm{O})(\mathrm{OH})_{2}$, MOF 3]. NMR analysis of MOF 3 digested with $\mathrm{D}_{3} \mathrm{PO}_{4}$ revealed up to $66 \%$ deprotection. MOF 1 was prepared according to a recently published procedure, and used as a control sorbent without the phosphorylurea group. Preparation of MOFs from organic linkers pre-functionalized with sorbent moieties affords precise loadings and facilitates investigation of sorption efficiency. Furthermore, the pendant phosphorylurea groups in these $\mathrm{UiO}$ frameworks line the channels and pores, and are ideally positioned to bind to $\mathrm{UO}_{2}{ }^{2+}$ and other actinides. Single-crystal X-ray diffraction results reported by Behrens demonstrate the presence of an amino group on the central aromatic ring of $\mathbf{L}_{\mathbf{1}}$ is too large to accommodate coplanar orientation of all three rings. The reported torsion angle of $47.9^{\circ}$ aligns the central ring orthogonally to the interior of the octahedral pore, directing the functional group into adjacent tetrahedral pores.

Powder X-Ray diffraction patterns for MOFs 1-3 are shown in Figure 15. The similarity between the simulated pattern for MOF 1 and the experimental patterns for MOFs 1-3 confirm the formation and maintenance of the UiO-68 phase, despite the fact that MOFs $\mathbf{2}$ and $\mathbf{3}$ were prepared using a bridging ligand containing a bulky functional group and by subsequent deprotection. Matching high angle peaks beyond $2 \theta$ of $30^{\circ}$ are observed for all samples, indicating highly crystalline structures for these $\mathrm{UiO}$ MOFs. SEM images indicated the formation of discrete octahedral crystals for all the three MOFs, with dimensions of $\sim 2-3 \mu \mathrm{m}$ per side (Figure 15). The thermogravimetric analysis of MOF 1-3 was consistent with possession of different functional groups. All TGA curves indicated distinct onset temperature for framework decomposition at $425{ }^{\circ} \mathrm{C}$ to form $\mathrm{ZrO}_{2}$. 
NEUP 11-3151

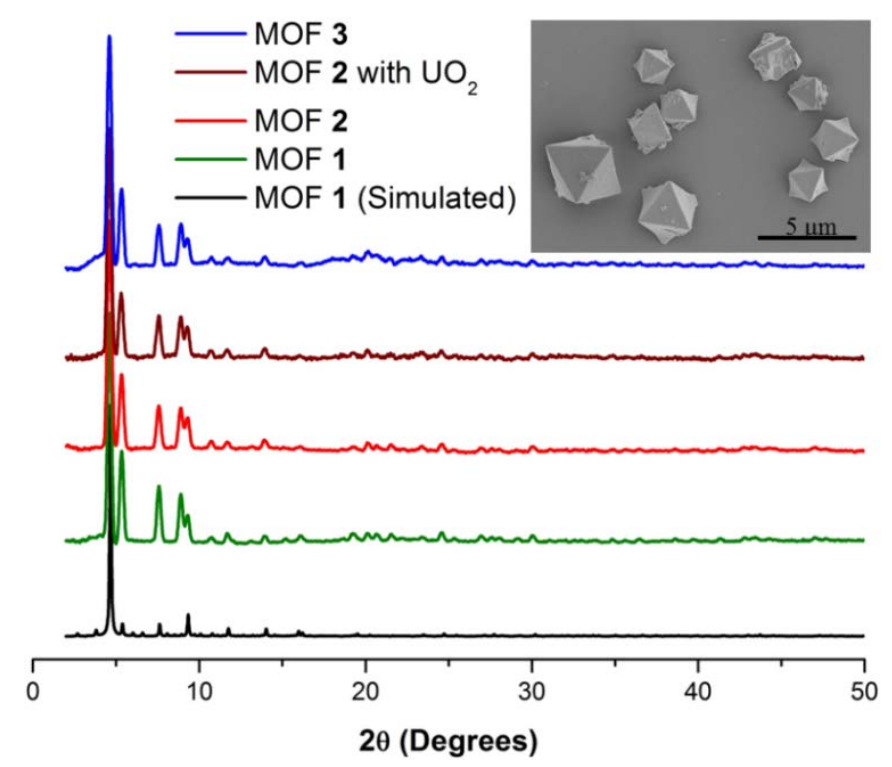

Figure 15. PXRD patterns for 1-3, simulated PXRD for 1, and PXRD for 2 after uranium sorption. Inset: representative SEM image for MOF 2.

Permanent porosity of the freeze-dried MOFs was demonstrated by $\mathrm{N}_{2}$ adsorption at $77 \mathrm{~K}$ (Figure 16). Type I isotherms were obtained for all three MOFs, with BET surface areas of 3730, 1350, and 750 $\mathrm{m}^{2} \cdot \mathrm{g}^{-1}$ for MOFs 1-3, indicative of microporous structure. Gas uptake simulations with Materials Studio were performed to yield BET surface areas of 2935, 2057, and $2521 \mathrm{~m}^{2} \mathrm{~g}^{-1}$, for 1-3, respectively. Calculations with PLATON yield 76.6\%, 65.7\%, and 68.4\% solvent accessible volume for MOFs 1-3, respectively. The surface area for MOF 3 was smaller than the ethoxy-protected precursor MOF 2, and both were significantly lower than value obtained by simulation. It is likely that evacuation of solvent from the MOFs caused minor framework distortion, reducing the surface area from the expected value. It is also possible the movement of pendant sorbent groups in MOF $\mathbf{2}$ and $\mathbf{3}$ are not well modeled by the simulation, causing a higher $\mathrm{N}_{2}$ uptake to be expected. Finally, investigation of MOF $\mathbf{3}$ by energy dissipative spectroscopy (EDS) revealed the presence of approximately $4 \mathrm{Br}$ per Zr cluster, accounting in part for the decrease in $\mathrm{N}_{2}$ uptake. 
NEUP 11-3151

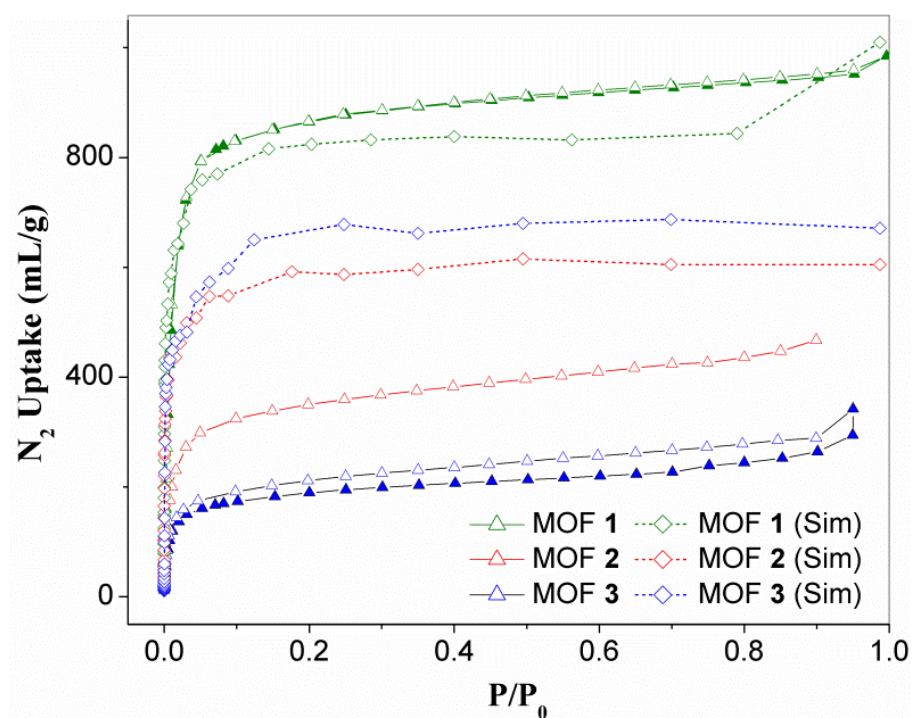

Figure 16. $\mathrm{N}_{2}$ uptake and simulated $\mathrm{N}_{2}$ uptake for MOFs 1-3 at 77K. Adsorption is represented with filled triangles, desorption with open triangles, and simulated results with open diamonds. Green, red, and blue colors represent sorption data for MOFs 1, 2, and 3, respectively.

Relative pore volume was further studied by measuring dye uptake by the MOFs. Freshly prepared MOFs 1-3 were thoroughly washed and soaked overnight in $24 \mathrm{mM}$ solutions of either Brilliant Blue (BBR-250) or Eosin Y dye. After rinsing with copious amounts of water to remove dye from the crystal surfaces, the MOFs were dried under vacuum and analyzed by TGA. MOF-1 demonstrated the greatest uptake (29.6 wt\% and $44.8 \mathrm{wt} \%$ for BBR-250 and Eosin Y, respectively), while MOF-2 did not adsorb a measurable amount of either dye. MOF-3 adsorbed $19.6 \mathrm{wt} \%$ BBR-250 and $10.0 \mathrm{wt} \%$ Eosin Y dye. The resulting pore volume hierarchy, MOF $1>$ MOF $3>$ MOF 2, is in strong agreement with simulated $\mathrm{N}_{2}$ uptake and solvent accessible volume calculated by PLATON. It is likely the dye molecules are capable of displacing $\mathrm{Br}$ from the channels of MOF 3, more accurately reflecting the relative pore volume of the MOF and indicating successful deprotection of the diethoxy groups.

Uranium sorption experiments were performed in water and simulated seawater at $\mathrm{pH} 2.5$, followed by analysis of the supernatant by UV-spectroscopy with the Arsenazo III dye, as described above. Confirmation of these measurements was obtained by ICP-MS. The sorbent was washed with $0.01 \mathrm{M}, 0.1 \mathrm{M}$ or $1.0 \mathrm{M} \mathrm{HCl}$ aqueous solutions to investigate $\mathrm{U}$ removal and determine the affinity of the ligand for the uranyl cation. Under these conditions MOF 1 exhibits no adsorption of U while MOFs 2 and 3 adsorb nearly all $U$ from solution (Figure 17). Analysis of the supernatant from $\mathrm{HCl}$ washes reveals that the sorbent has strong affinity for $U$. After washing with $0.01 \mathrm{M} \mathrm{HCl}$, the amount of $\mathrm{U}$ remaining in the sorbent is statistically unchanged, while 31-66\% is eluted by washing with $0.1 \mathrm{M} \mathrm{HCl}$. Notably, some $\mathrm{U}$ remains in the sorbent, even after washing with $1 \mathrm{M} \mathrm{HCl}$. Amidoxime fibers were used as control samples, which showed nearly complete desorption of bound uranium by washing with $0.1 \mathrm{M}$ $\mathrm{HCl}$, suggesting physisorption on the surface of the polymer material as the primary mode of $\mathrm{U}$ binding. 

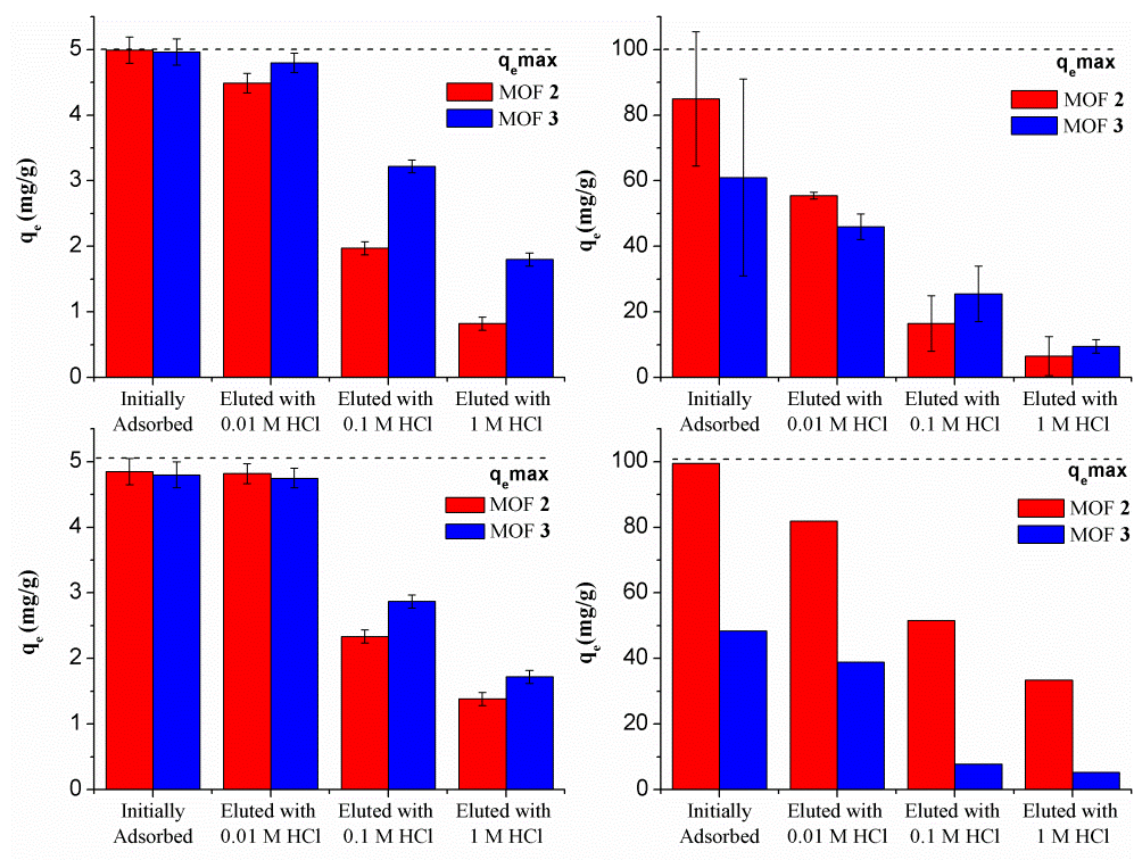

Figure 17. Sorption and desorption of uranium with MOFs 2 and 3, with $[\mathrm{U}]_{0}=5 \mathrm{ppm}$ and $\mathrm{pH}=2.5$ in water (top left) and simulated seawater (bottom left), and with $[\mathrm{U}]_{0}=100 \mathrm{ppm}$ at $\mathrm{pH} 2.5$ in simulated seawater analyzed by UV spectroscopy (top right) and ICP-MS (bottom right). Sorption capacity $\left(q_{e}\right)$ denotes the quantity of uranium extracted by the MOF, as determined by measuring the amount of uranium remaining in the supernatant. Measurements of $q_{e}$ were made after the initial period of adsorption and following elution with $\mathrm{HCl}$ washes.

Sorption isotherms at $\mathrm{pH} 2.5$ and 5 were measured to determine saturation capacity for the MOFs, with the obtained results fit to the Langmuir model, discussed previously. At $\mathrm{pH} 2.5$, saturation capacities of $217 \mathrm{mg} \mathrm{U} / \mathrm{g}$ in water and $188 \mathrm{mg} \mathrm{U} / \mathrm{g}$ in simulated seawater were obtained for 2 . By comparison, amidoxime resin examined at $\mathrm{pH} 2$ in sodium carbonate salt solution possessed saturation capacity of $54 \mathrm{mg} \mathrm{U} / \mathrm{g}$. Saturation capacity for 3 is lower in the both media, at 109 and $32 \mathrm{mg} \mathrm{U} / \mathrm{g}$ sorbent for water and artificial seawater, respectively. At pH 5, saturation capacities of 152 and $104 \mathrm{mg}$ U/g were obtained for $\mathbf{2}$ and $\mathbf{3}$ in water. Isotherm data are displayed in Figure 18.

Determination of sorption efficiency requires knowledge of the binding modes for $\mathrm{UO}_{2}{ }^{2+}$ with the phosphorylurea sorbent moiety. We performed density functional theory (DFT) calculations to assess optimized geometries and relative stabilities for a series of $\mathrm{UO}_{2}{ }^{2+}$ complexes with carbamoylphosphoramidic acid, representative of the sorbent in MOFs $\mathbf{2}$ and $\mathbf{3}$.

Recent publications suggest the possibility of several binding motifs for $\mathrm{UO}_{2}{ }^{2+}$ complexes. The related CMPO ligand is known to coordinate actinides in a monodentate fashion through the phosphoryl oxygen, as has been determined through experimental and computational studies. Similarly, the carbonyl or phosphoryl oxygen could bind $\mathrm{UO}_{2}{ }^{2+}$ to afford binding motifs I and II. Alternatively, both oxygen atoms could coordinate in a bidentate fashion, resulting in motif III. These binding motifs are displayed in Figure 19. 

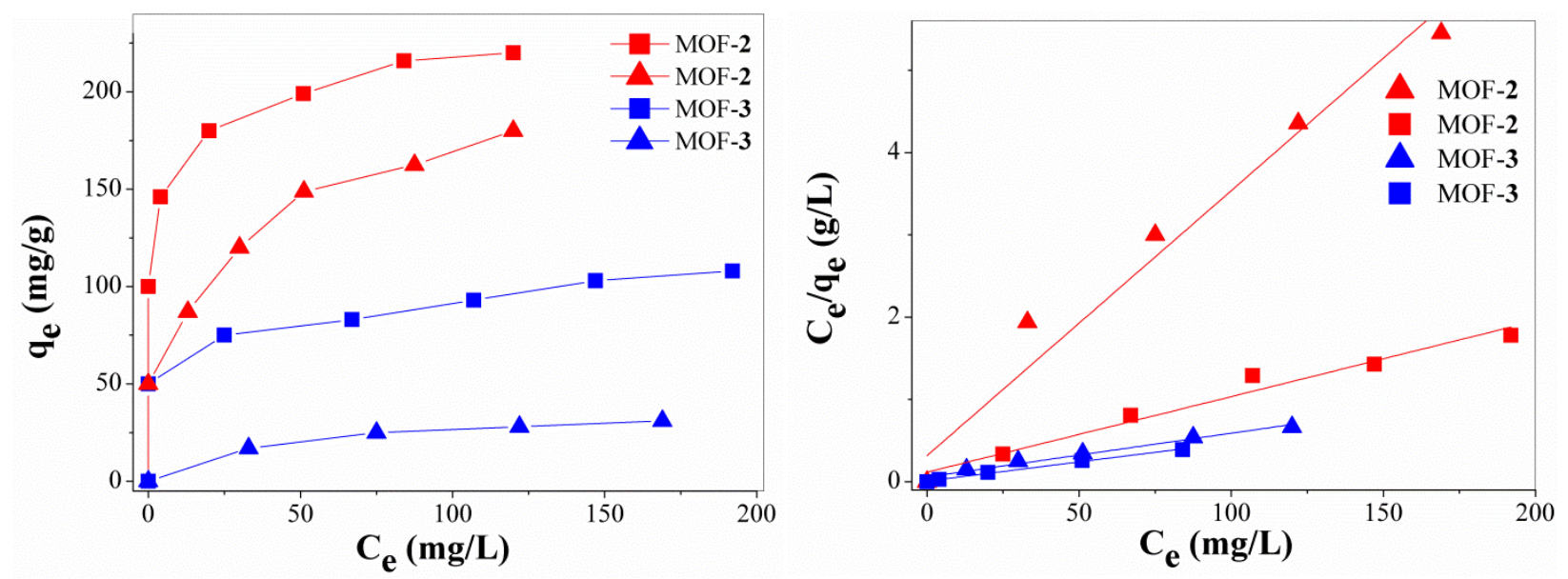

Figure 18. (Left) Langmuir adsorption isotherms for MOFs 2 and $\mathbf{3}$ at pH 2.5. Sorption capacities $\left(q_{e}\right)$ in water and simulated seawater are displayed by squares and triangles, respectively, and plotted against the $\mathrm{U}$ concentration at equilibrium. (Right) Linear expression of the Langmuir isotherm. Values for saturation sorption capacity $\left(q_{\max }\right)$, and the Langmuir constant $\left(K_{L}\right)$ can be obtained from the slope and yintercept.

Examinations of the structural models for MOFs 2-3 reveal inter-sorbent distances of approximately 4.5 - $4.8 \AA$ between carboxyl and phosphoryl oxygen. The large coordination sphere of actinides in conjunction with appropriate inter-atomic distances for sorbents indicates two phosphorylurea ligands could bind the same $\mathrm{UO}_{2}{ }^{2+}$ cooperatively. Consideration of binding interactions with two ligands increases possible motifs to include two monodentate carbonyl oxygen (I-I), two monodentate phosphoryl oxygen (II-II), one monodentate carbonyl oxygen and one monodentate phosphoryl oxygen (I-II), one monodentate oxygen and one bidentate ligand (I-III and II-III for carbonyl or phosphoryl oxygen, respectively), or two bidentate ligands (III-III).
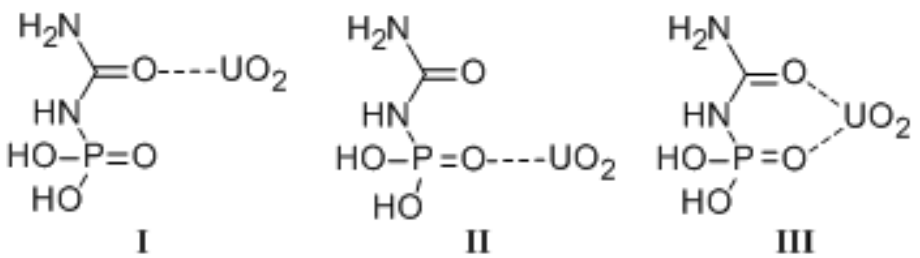

Figure 19. Three uranyl binding motifs for carbamoylphosphoramidic acid investigated by DFT calculations: uranyl bound to carbonyl oxygen (I), uranyl bound to phosphoryl oxygen (II), and bidentate uranyl coordination (III).

$\mathrm{UO}_{2}{ }^{2+}$ is known to form a pentahydrate $\left[\mathrm{UO}_{2}\left(\mathrm{H}_{2} \mathrm{O}\right)\right]^{2+}$ complex when solvated, and all coordination sites on $\mathrm{UO}_{2}{ }^{2+}$ not interacting with a sorbent were filled with aqua ligands. While calculations were also performed in the gas phase, discussion focuses on the solvated complex. Bond lengths for geometry optimized $\left[\mathrm{UO}_{2}\left(\mathrm{H}_{2} \mathrm{O}\right)\right]^{2+}$ were in good agreement with both EXAFS data and recently published computational results.

The $\mathrm{pK}_{\mathrm{a}}$ for the model ligand was approximated using the $\mathrm{pK}_{\mathrm{a}}$ of phosphoric acid $\left(\mathrm{pK}_{\mathrm{a}}=2.12\right)$, indicating $60 \%$ of the ligand would be deprotonated at the $\mathrm{pH}$ of the uranium sorption experiments. Geometry optimization calculations were performed for all binding motifs with both protonated and 
deprotonated ligands (deprotonated ligand systems are denoted by an asterisk). Frequency calculations were performed for geometrically optimized structures to obtain thermodynamic data. Representative sturctures are displayed in Figure 20.
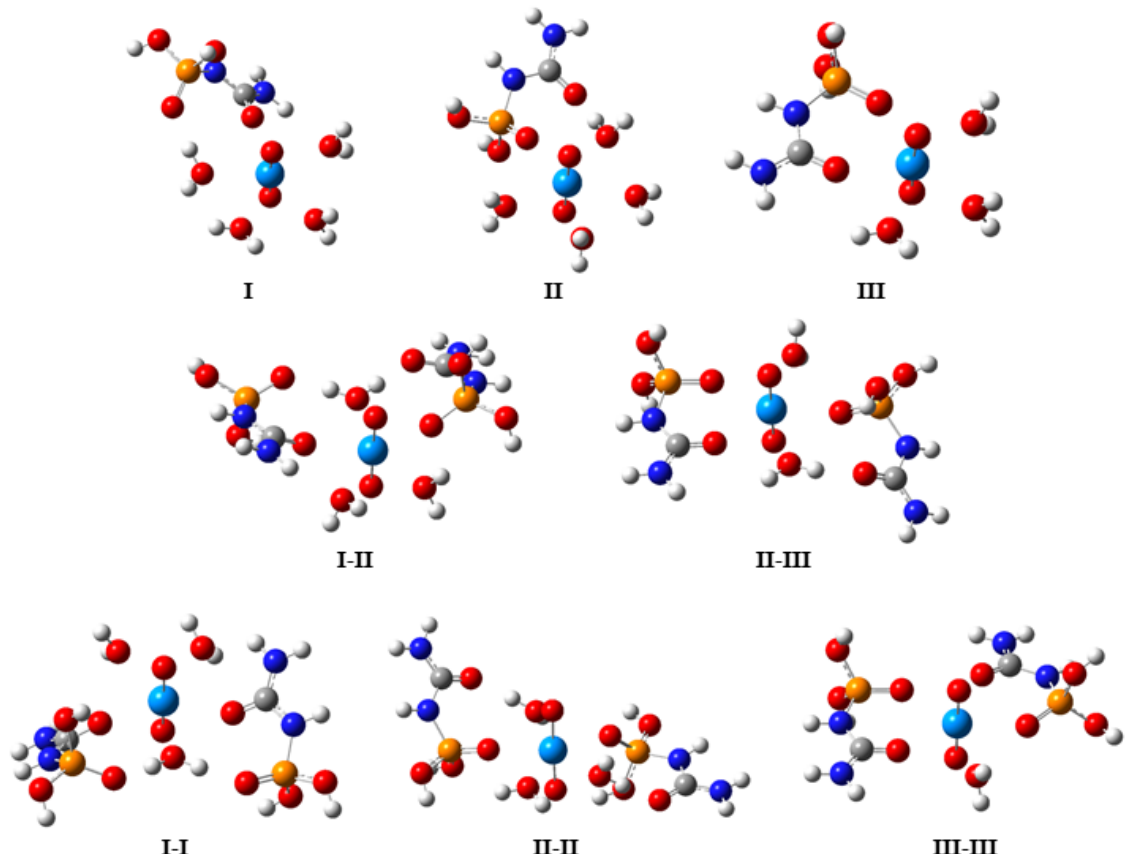

Figure 20. Representative structures with optimized binding geometries for the coordination of $\mathrm{UO}_{2}{ }^{2+}$ to one and two ligands. In all instances, $\mathrm{UO}_{2}{ }^{2+}$ maintains five coordination sites around the equatorial plane. Complexes formed with deprotonated ligands adopted identical structures. Uranium, carbon, nitrogen, oxygen, phosphorus, and hydrogen are light blue, grey, dark blue, red, orange, and white, respectively.

Reaction enthalpies were calculated from the difference between the heat of formation for products and reactants, obtained from frequency calculations of optimized structures. The most enthalpically favored binding motif was II-II, coordination by two fully protonated ligands through the phosphoryl oxygen. Coordination motifs I-II and I-I, where phosphoryl oxygen are replaced by one and two carboxyl oxygen, were only slightly $\left(<1 \mathrm{kcal} \mathrm{mol}^{-1}\right)$ less favorable. In general, binding with two ligands was better than one ligand, and motifs with protonated ligands were enthalpically preferred to motifs with deprotonated ligands. The preference for protonated ligands is confirmed by the observed decrease in $\mathrm{UO}_{2}{ }^{2+}$ sorption by 2 at $\mathrm{pH}$, and the calculated binding enthalpies are in good agreement with recently published values for $\mathrm{UO}_{2}{ }^{2+}$ with $n$-octyl(phenyl) CMPO and diphenyl CMPO. Thermodynamic data are summarized in Table 10, where $\Delta \mathrm{H}_{\mathrm{aq}}$ refers to the reaction enthalpy in the aqueous phase.

For motif II-II, $\mathrm{UO}_{2}{ }^{2+}$ was located $2.36 \AA$ and $2.38 \AA$ from the two coordinating phosphoryl oxygen. The oxygen atom of the three aqua ligands were 2.44, 2.46, and $2.49 \AA$ from $\mathrm{U}$, and $\mathrm{U}=\mathrm{O}$ bond lengths were $1.76 \AA$. Bond distances for coordinating oxygen were similar for I-I and I-II. Bond lengths contracted slightly upon deprotonation, as shown by distances of $2.33 \AA$ and $2.35 \AA$, and $2.32 \AA$ and 2.34 $\AA$ for II-II* and I-I*, respectively. Distances from $\mathrm{UO}_{2}{ }^{2+}$ to the aqua ligands and $\mathrm{U}=\mathrm{O}$ bonds remained unchanged.

Natural Bond Orbital (NBO) analysis was performed for motifs I-I, II-II, I-II, III-III, II-III, IIII*, III-III*, and II-III*. The Wiberg bond indices for all structures gives $(\mathrm{R}=) \mathrm{O}-\mathrm{U}$ bond order $(\mathrm{R}=\mathrm{P}$ or $\mathrm{C}$ ) between 0.4486 and 0.6292 . NBO analysis indicates the $(\mathrm{R}=) \mathrm{O}-\mathrm{U}$ bonds to be $89-92 \%$ localized 
on the phosphoryl oxygen, indicating that the bonding interactions between $\mathrm{UO}_{2}{ }^{2+}$ and the ligands have significant ionic character. Covalent character is also evident, as can be seen from localized MOs for complexes II and II-II, which clearly display a (P=)O - U bonding interaction, shown in Figure 21.
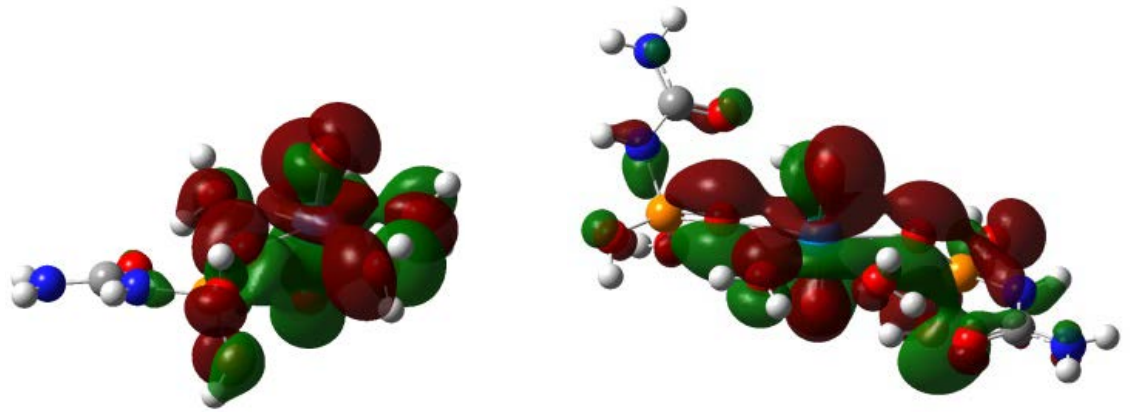

Figure 21. Localized molecular orbitals for structures II (left) and II-II (right) displaying interaction between $\mathrm{U}$ and the phosphoryl oxygen, indicating the binding of $\mathrm{UO}_{2}{ }^{2+}$ is partially covalent in character. HOMO - 7 is displayed for II, and HOMO - 13 for II-II.

Table 10. Stability data for $\left[\mathrm{UO}_{2}(\mathrm{~L})_{x}\left(\mathrm{OH}_{2}\right)_{y}\right]^{2}$ complexes as determined by DFT calculations

\begin{tabular}{|c|c|c|c|}
\hline Motif & $\begin{array}{c}\text { Charge per } \\
\text { Ligand }\end{array}$ & $\begin{array}{c}\Delta \mathrm{H}_{\mathrm{aq}} \\
(\mathrm{kcal} / \mathrm{mol})\end{array}$ & $\begin{array}{c}\Delta \Delta \mathrm{H}_{\mathrm{aq}} \\
(\mathrm{kcal} / \mathrm{mol})\end{array}$ \\
\hline I & --- & -146.06 & 51.87 \\
\hline II & --- & -145.87 & 52.06 \\
\hline III & --- & -2.57 & 195.36 \\
\hline & & & \\
\hline I-I & --- & -197.54 & 0.39 \\
\hline I-II & --- & -197.83 & 0.09 \\
\hline I-III & --- & -175.94 & 21.99 \\
\hline II-II & --- & -197.93 & 0.00 \\
\hline II-III & --- & -175.17 & 22.75 \\
\hline III-III & --- & -1.89 & 196.04 \\
\hline & & & \\
\hline I* $^{*}$ & -1 & -22.29 & 175.64 \\
\hline II* $^{*}$ & -1 & -26.18 & 171.75 \\
\hline III* $^{*}$ & -1 & -23.92 & 174.01 \\
\hline I-I* $^{*}$ & -1 & -41.83 & 156.10 \\
\hline I-II* $^{*}$ & -1 & -34.77 & 163.16 \\
\hline I-III* & -1 & -43.33 & 154.60 \\
\hline II-II* & -1 & -47.69 & 150.23 \\
\hline II-III* $^{*}$ & -1 & -49.98 & 147.95 \\
\hline III-III* $^{*}$ & -1 & -42.91 & 155.01 \\
\hline
\end{tabular}

The computational results support the experimental data and provide insight to the observed sorption capacities. Coordination of $\mathrm{UO}_{2}{ }^{2+}$ by protonated carbamoylphosphoramidic acid was calculated to be approximately $150 \mathrm{kcal} / \mathrm{mol}$ more favorable than coordination by the deprotonated carbamoylphosphoramidate analog. Estimating the $\mathrm{pK}_{\mathrm{a}}$ for the deprotected MOF-3 to be similar to 
phosphoric acid, approximately $60 \%$ of the ligands are expected to be deprotonated at $\mathrm{pH} 2.5$, and thus less thermodynamically inclined to bind $\mathrm{UO}_{2}{ }^{2+}$. It is worth noting that saturation capacity of MOF-3 in water is $50 \%$ of the ethoxy-protected MOF-2, which is not deprotonated under those conditions. Sorption studies at pH 5 confirm this trend, where decreased saturation capacities were observed for MOFs 2 and 3.

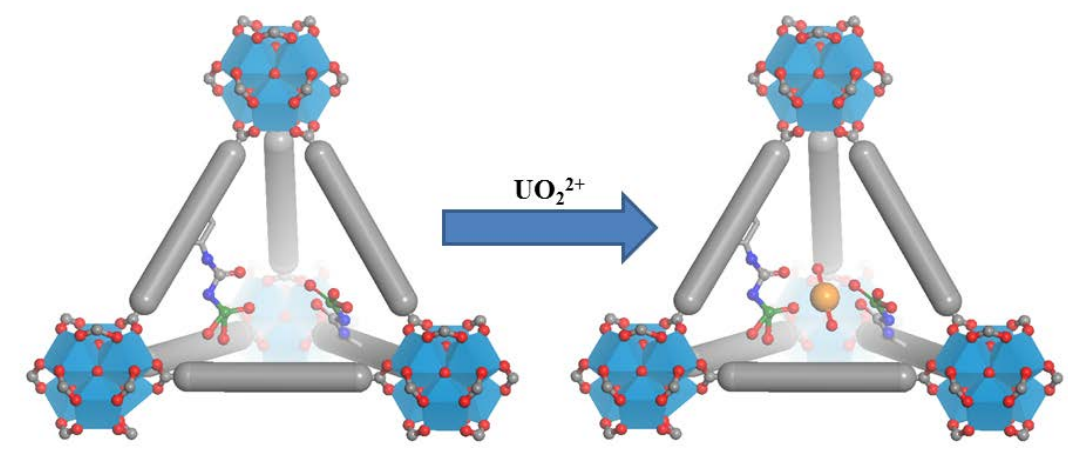

Figure 22. Simplified schematic depicting the uranyl-binding pocket formed in the tetrahedron of the MOFs. $\mathrm{UO}_{2}{ }^{2+}$ is coordinated in a monodentate fashion to the phosphoryl oxygen. Distances between oxygen range from $4.5-4.8 \AA$, accommodating U-O bond lengths appropriate for binding motif II-II.

Application of the preferred binding motif to the observed saturation capacities for MOFs 2-3 allowed determination of maximum sorbent efficiency. All sorbent sites on MOF-2 were saturated, binding $1 \mathrm{U}$ for every 2 sorbent groups in water and 2.3 sorbent groups in seawater. In contrast, MOF-3 absorbed $1 \mathrm{U}$ per 4.5 or 15.3 sorbent groups in water and seawater, respectively. Uranyl extraction is facilitated by the cooperative binding of the phosphoryl groups that are located inside tetrahedral cavities and have suitable inter-sorbent distances (Figure 22). U extraction efficiencies can be further improved by using MOFs with optimal channel structures and incorporating sorbent ligands with higher affinity for actinides, while further tuning of MOF morphology may provide a unique means to selectively extract $\mathrm{UO}_{2}{ }^{2+}$ over competing ions prevalent in seawater.

\section{Elucidate effects of chelating amidoxime ligands on uranyl extraction}

Preliminary studies on the binding of amidoxime to uranyl were undertaken by density-functional theory (DFT) calculations. While these previous studies have demonstrated how amidoxime coordinates to uranyl, questions remain as to why it exhibits such a strong affinity, and more importantly, how to improve upon the amidoxime functional group for uranium sequestration. It is anticipated improving the $\mathrm{UO}_{2}{ }^{2+}$ binding strength of the sorbent as well as development of well-designed multi-topic chelators would significantly improve the properties of sorbent materials. Several studies have revealed the tridentate chelating cyclic imide dioxime ligand to bond more strongly than the open chain diamidoxime, and complementary geometry for amidoxime-decorated hosts has very recently been investigated through de novo structure-based computational screening. However, detailed investigations of amidoxime-uranyl binding interactions have been largely neglected. We performed DFT calculations to probe the structural, electronic, and thermochemical properties for a series of amidoxime-derived uranyl complexes. We observed bond strength correlates directly with the resonance electron donating properties of the substituent adjacent to the uranyl-binding oximate group. From these observations, we were able to propose two new promising amidoxime-derived sorbents for further investigation in extracting $\mathrm{UO}_{2}{ }^{2+}$ from seawater. 


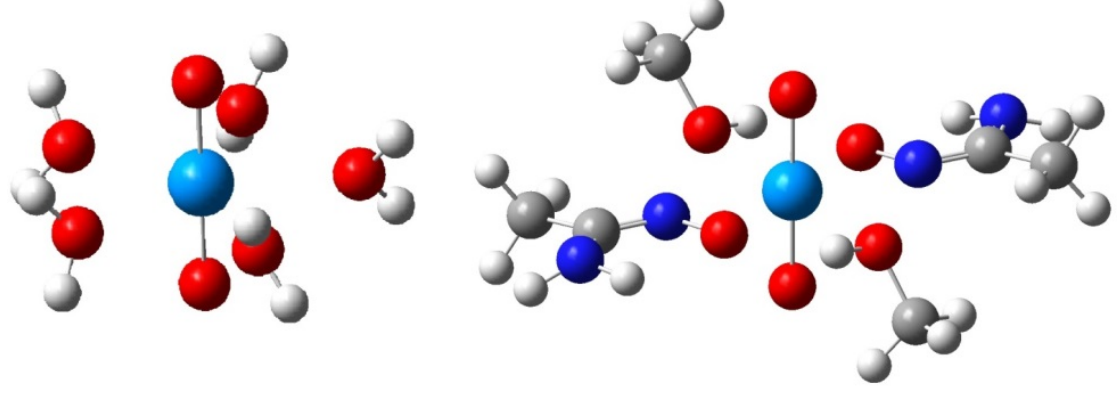

Figure 23. Optimized structures for $\left[\mathrm{UO}_{2}\left(\mathrm{H}_{2} \mathrm{O}\right)_{5}\right]^{2+}$ (left) and $\left[\mathrm{UO}_{2}(\mathrm{AO})_{2}(\mathrm{MeOH})_{2}\right]$ (right), with the latter displaying $\eta^{2}$ binding of amidoximate (AO) to uranyl. Red, white, grey, dark blue, and light blue spheres represent $\mathrm{O}, \mathrm{H}, \mathrm{C}, \mathrm{N}$, and $\mathrm{U}$, respectively. An identical color palate is used in all subsequent figures.

The reliability of the proposed theoretical method was tested first by gas phase calculations on uranyl pentahydrate $\left[\mathrm{UO}_{2}\left(\mathrm{H}_{2} \mathrm{O}\right)_{5}\right]^{2+}$ and uranyl diacetamidoximate with two coordinated methanol molecules $\left[\mathrm{UO}_{2}(\mathrm{AO})_{2}(\mathrm{MeOH})_{2}\right](\mathrm{AO}=$ acetamidoximate), shown in Figure 23. EXAFS measurements of $\left[\mathrm{UO}_{2}\left(\mathrm{H}_{2} \mathrm{O}\right)_{5}\right]^{2+}$ and bond lengths obtained by single crystal X-ray analysis of $\left[\mathrm{UO}_{2}(\mathrm{AO})_{2}(\mathrm{MeOH})_{2}\right]$ reveal that the computational method reliably predicted the structural parameters (average errors of $0.062 \AA$ and $0.049 \AA$, respectively). For both systems, bond lengths were generally over-estimated with the largest discrepancies for the equatorially coordinated solvent molecules. Average distances for U-solvent coordination differed from experimental results by $0.082 \AA$ and $0.142 \AA$ for $\left[\mathrm{UO}_{2}\left(\mathrm{H}_{2} \mathrm{O}\right)_{5}\right]^{2+}$ and $\left[\mathrm{UO}_{2}(\mathrm{AO})_{2}(\mathrm{MeOH})_{2}\right]$. All other structural discrepancies were less than $0.05 \AA$ in deviation.

In an effort to obtain more accurate structural values, the calculation for $\left[\mathrm{UO}_{2}\left(\mathrm{H}_{2} \mathrm{O}\right)_{5}\right]^{2+}$ was repeated with solvation effects taken into consideration as experimental values for $\left[\mathrm{UO}_{2}\left(\mathrm{H}_{2} \mathrm{O}\right)_{5}\right]^{2+}$ was obtained by EXAFS while in water. Consideration of solvent effects improved the predicted structural parameters relative to the gas phase calculations, with average errors decreasing to $0.036 \AA$. The influence of solvation significantly altered the axial $\mathrm{U}=\mathrm{O}$ distance for $\left[\mathrm{UO}_{2}\left(\mathrm{H}_{2} \mathrm{O}\right)_{5}\right]^{2+}$, bringing the bond length within $0.002 \AA$ of the experimentally determined value, and brought the U-solvent distance to within $0.05 \AA$ of the experimental value. Selected average bond lengths are displayed in Table 11.

The mother liquor used for the crystal preparation of $\left[\mathrm{UO}_{2}(\mathrm{AO})_{2}(\mathrm{MeOH})_{2}\right]$ was a mixture of unspecified volumes of methanol, nitromethane, and dichloroethane, making meaningful consideration of solvent effects difficult. Calculations of $\left[\mathrm{UO}_{2}(\mathrm{AO})_{2}(\mathrm{MeOH})_{2}\right]$ were performed including effects of solvation in methanol, yielding a smaller deviation in the U-solvent distance than calculations performed in the gas phase and average error of $0.042 \AA$. These bond lengths are displayed in Table 1 . Similar calculations performed with dichloroethane and nitromethane further improved the U-solvent distance, but at the expense of the amidoximate $\mathrm{O}-\mathrm{N}$ bond length, resulting in average errors of $0.068 \AA$ and 0.067 $\AA$, respectively. Similar bond lengths and average errors were obtained from varying the dielectric coefficient using water as the solvent, or from averaging the dielectric coefficients for methanol, dichloroethane, and nitromethane.

In aqueous solutions, $\mathrm{UO}_{2}{ }^{2+}$ is coordinated by five aquo ligands in the equatorial plane. This has been demonstrated both computationally and experimentally, though prior studies also demonstrate a proclivity to hexacoordination in the gas phase. Previous work performed on AO-uranyl complexes demonstrates $\eta^{2}$ coordination displaces two equatorial aquo ligands to obtain the thermodynamically 
favored structure. Calculations were performed for one $\mathrm{AO}$ ligand coordinating to $\mathrm{UO}_{2}{ }^{2+}$, with aquo ligands completing the inner coordination sphere.

Table 11. Selected Average Bond Lengths $(\AA)$ for $\left[\mathrm{UO}_{2}\left(\mathrm{H}_{2} \mathrm{O}\right)_{5}\right]^{2+}$ and $\left[\mathrm{UO}_{2}(\mathrm{AO})_{2}(\mathrm{MeOH})_{2}\right]$

\begin{tabular}{|c|c|c|c|c|c|}
\hline$\left[\mathrm{UO}_{2}\left(\mathrm{H}_{2} \mathrm{O}\right)_{5}\right]^{2+}$ & & \multicolumn{2}{|c|}{ Gas Phase } & \multicolumn{2}{|c|}{ Solvated } \\
\hline & Experimental $^{\mathrm{a}}$ & Calculated & $\Delta$ & Calculated & $\Delta$ \\
\hline $\mathrm{U}=\mathrm{O}$ & 1.76 & 1.748 & 0.012 & 1.758 & 0.002 \\
\hline $\mathrm{U}-\mathrm{OH}_{2}$ & 2.41 & 2.492 & 0.082 & 2.460 & 0.050 \\
\hline Avg. Error & & & 0.062 & & 0.036 \\
\hline$\left[\mathrm{UO}_{2}(\mathrm{AO})_{2}(\mathrm{MeOH})_{2}\right]$ & & \multicolumn{2}{|c|}{ Gas Phase } & \multicolumn{2}{|c|}{ Solvated $(\mathrm{MeOH})$} \\
\hline & Experimental $^{\mathrm{b}}$ & Calculated & $\Delta$ & Calculated & $\Delta$ \\
\hline $\mathrm{U}=\mathrm{O}$ & 1.789 & 1.789 & 0.000 & 1.806 & 0.017 \\
\hline U-OMe & 2.458 & 2.600 & 0.142 & 2.579 & 0.121 \\
\hline U-N & 2.398 & 2.440 & 0.042 & 2.429 & 0.031 \\
\hline $\mathrm{U}-\mathrm{O}$ & 2.383 & 2.357 & 0.026 & 2.334 & 0.049 \\
\hline $\mathrm{O}-\mathrm{N}$ & 1.409 & 1.369 & 0.040 & 1.378 & 0.031 \\
\hline $\mathrm{C}=\mathrm{N}$ & 1.290 & 1.288 & 0.002 & 1.292 & 0.002 \\
\hline Avg. Error & & & 0.049 & & 0.042 \\
\hline
\end{tabular}

a. Experimental data from literature. b. Experimental data from literature.

The valence orbitals for $\mathrm{UO}_{2}{ }^{2+}$ in aqueous solution are $\sigma(\mathrm{f}), \pi(\mathrm{f}), \sigma(\mathrm{d})$, and $\pi(\mathrm{d})$ at $-11.1,-11.9$, 12.2, and $-12.2 \mathrm{eV}$. A recent DFT study of the uranyl-peroxo system revealed the $\sigma$ and $\pi$ bonding orbitals of a simple peroxo ligand to be lower in energy than the uranyl $\sigma(d)$ and $\pi(d)$, with covalent interactions occurring between the peroxo lone pair orbitals and the uranyl $5 f$ orbitals. As the oximate function is partly composed of two adjacent $\pi$-conjugated Lewis bases, similar to the peroxo ligand, we expected similar electronic structure in uranyl-AO complexes. However, the incorporation of the adjacent amine and methyl group result in electron donation, raising the energy level of the oximate orbitals. While the $\sigma$ bonding orbitals of $\mathrm{N}-\mathrm{O}$ have no contribution to bonding between the oximate function and uranium, similar to peroxide, the $\pi$ bonding orbitals were observed to be higher in energy than the uranyl $\sigma(\mathrm{d})$ and $\pi(\mathrm{d})$ and thus had significant bonding interactions. Most notably, the conjugation of O-N $\pi$ - orbitals overlap constructively with the $1 \pi_{\mathrm{g}}$ and $2 \pi_{\mathrm{u}}$ orbitals of $\mathrm{UO}_{2}{ }^{2+}$. Other major bonding contributions come from interaction between the lone pairs on oxygen and nitrogen with the uranium $5 f$ orbital $1 \varphi_{\mathrm{u}}$, the oxygen lone pair with $\mathrm{UO}_{2}{ }^{2+} 3 \sigma_{\mathrm{u}}$, and the oxygen lone pair and $\mathrm{C}=\mathrm{N} \pi$-bonding orbital with the 5 f orbital $1 \delta_{\mathrm{u}}$. Several important orbital interactions are displayed in Figure 24, and MO energies and character are summarized in Table 12.

To obtain further insight into the bonding nature of the uranyl-AO complex, Wiberg Bond Indices (WBIs) were calculated using natural bond orbital (NBO) analysis. WBI values between 0.1 and 0.5 are regarded as largely ionic, while covalent bonds have WBIs with values in the vicinity of 1 . For example, WBIs for bonds between $\mathrm{UO}_{2}{ }^{2+}$ and phosphine oxides used in nuclear fuel reprocessing were calculated to be below 0.393, indicative of ionic bonding and electrostatic interactions, while a phosphoryl-urea complex used to extract $\mathrm{UO}_{2}{ }^{2+}$ from simulated seawater yielded a higher WBI of 0.629 , suggestive of some covalent character. These phosphine-based ligands often coordinate through the phosphoryl oxygen 
in a monodentate fashion, yielding only one WBI value. In contrast, AO binds through both the oximate $\mathrm{O}$ and N, yielding WBIs of 0.885 and 0.576, respectively, and a combined WBI of 1.461.

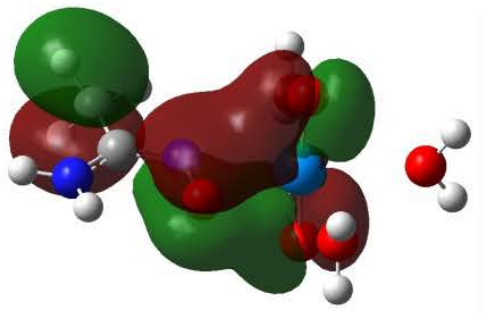

44: $\pi(\mathrm{O}-\mathrm{N}) / 1 \pi_{\mathrm{g}}$

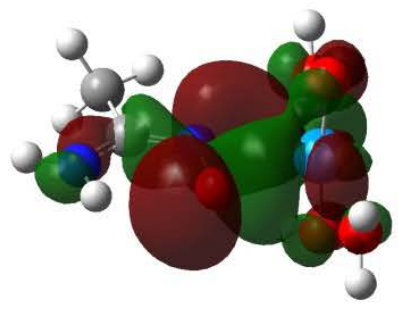

57: $\mathrm{O}_{\mathrm{LP}}, \mathrm{N}_{\mathrm{LP}} / 1 \varphi_{\mathrm{u}}$

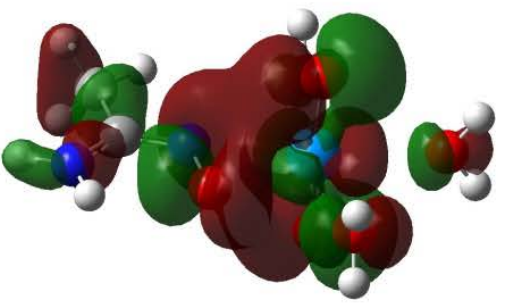

54: $\pi(\mathrm{O}-\mathrm{N}) / 2 \pi_{\mathrm{u}}$

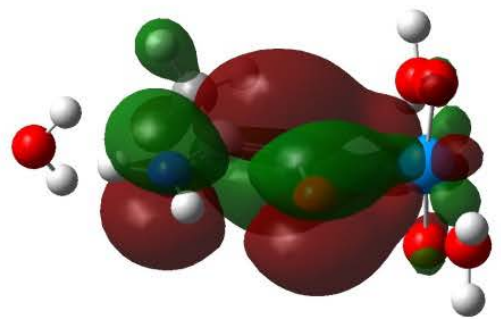

58: $\mathrm{O}_{\mathrm{LP}}, \pi(\mathrm{C}=\mathrm{N}) / 1 \delta_{\mathrm{u}}$

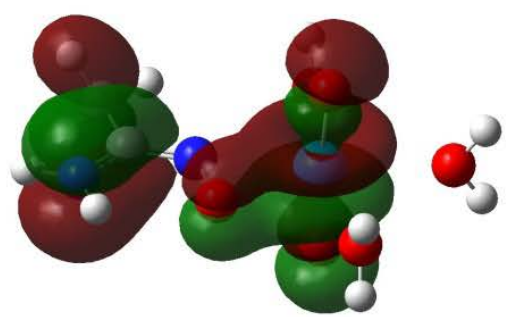

55: $\mathrm{O}_{\mathrm{LP}} / 3 \sigma_{\mathrm{u}}$

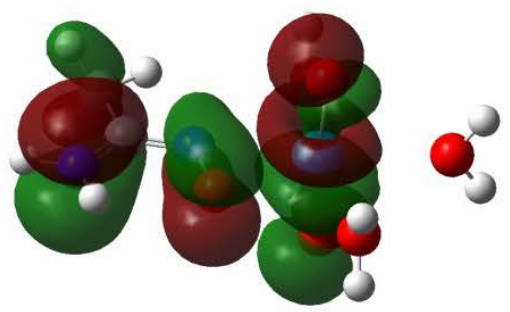

56: $\pi(\mathrm{O}-\mathrm{N}) / 3 \sigma_{\mathrm{u}}$

Figure 24. Select molecular orbitals for $\left[\mathrm{UO}_{2}(\mathrm{AO})\left(\mathrm{H}_{2} \mathrm{O}\right)_{3}\right]^{+1}$. Subscript LP represents a lone pair for orbitals 55, 57, and 58. Orbitals 44, 54, 55, 57, and 58 comprise the majority of bonding interactions between AO and uranyl. The absence of bonding in 56 between $\pi(\mathrm{O}-\mathrm{N})$ and $3 \sigma_{\mathrm{u}}$ is due to symmetry considerations, which is similar for orbital 50 (not shown). Geometries were optimized with solvation effects.

Table 12. Energies and Characters of the MOs for $\left[\mathrm{UO}_{2}(\mathrm{AO})\left(\mathrm{H}_{2} \mathrm{O}\right)\right]^{+1}$

\begin{tabular}{|c|c|c|}
\hline MO & Energy $(\mathrm{eV})$ & Character \\
\hline 58 & -9.330 & $\mathrm{O}_{\mathrm{LP}}, \pi(\mathrm{C}=\mathrm{N}) / 1 \delta_{\mathrm{u}}$ \\
\hline 57 & -11.185 & $\mathrm{O}_{\mathrm{LP}}, \mathrm{N}_{\mathrm{LP}} / 1 \varphi_{\mathrm{u}}$ \\
\hline 56 & -11.618 & $\pi(\mathrm{O}-\mathrm{N}) / 3 \sigma_{\mathrm{u}}$ \\
\hline 55 & -12.418 & $\mathrm{O}_{\mathrm{LP}} / 3 \sigma_{\mathrm{u}}$ \\
\hline 54 & -12.747 & $\pi(\mathrm{O}-\mathrm{N}) / 2 \pi_{\mathrm{u}}$ \\
\hline 53 & -13.142 & $2 \pi_{\mathrm{u}}$ \\
\hline 52 & -13.435 & $2 \pi_{\mathrm{u}}$ \\
\hline 51 & -13.466 & $2 \pi_{\mathrm{u}}$ \\
\hline 50 & -13.524 & $\pi(\mathrm{O}-\mathrm{N}-\mathrm{C}-\mathrm{N}) / 1 \pi_{\mathrm{g}}$ \\
\hline 49 & -13.726 & $3 \sigma_{\mathrm{g}}$ \\
\hline 48 & -13.794 & $3 \sigma_{\mathrm{g}}$ \\
\hline 47 & -14.011 & $1 \pi_{\mathrm{g}}$ \\
\hline 46 & -14.220 & $\mathrm{O}_{\mathrm{LP}}, \pi(\mathrm{N}-\mathrm{C}-\mathrm{N}) / 3 \sigma_{\mathrm{g}}$ \\
\hline 45 & -14.404 & $\sigma\left(\mathrm{H}_{3} \mathrm{C}-\mathrm{C}\right)$ \\
\hline 44 & -14.411 & $\pi(\mathrm{O}-\mathrm{N}) / 1 \pi_{\mathrm{g}}$ \\
\hline
\end{tabular}

NBO analysis revealed bonds between $\mathrm{U}$ and hybridized orbitals from light elements were $85.6 \%$ $\mathrm{O}$ and $86.1 \% \mathrm{~N}$ character, while the interaction between $\mathrm{U}$ and the $\mathrm{O}$ p-orbital was $92.8 \%$ localized on $\mathrm{O}$. The localization of these orbitals suggests that modulating energy levels could result in more equal 
electron contribution and a stronger bond. A conjugated system of $\pi$ orbitals connects the oxime function to the adjacent amine, providing a means for electron donation through mesomeric effects. This observation lead us to speculate uranyl-amidoximate bond strength could be tuned by varying the adjacent substituent. By increasing electron donation, the energy level of the bonding MOs should increase to afford a greater contribution from uranyl and to yield a more thermodynamically stable bond. To investigate this hypothesis, we calculated the thermodynamics for a series of oximate-derived ligands coordinated to $\mathrm{UO}_{2}{ }^{2+}$ and probed the bonding characteristics by $\mathrm{NBO}$ analysis. Adjacent substituents on the oximate-based ligands were selected on the basis of their electron donating character via resonance. The series of ligands is displayed in the inset of Figure 25.

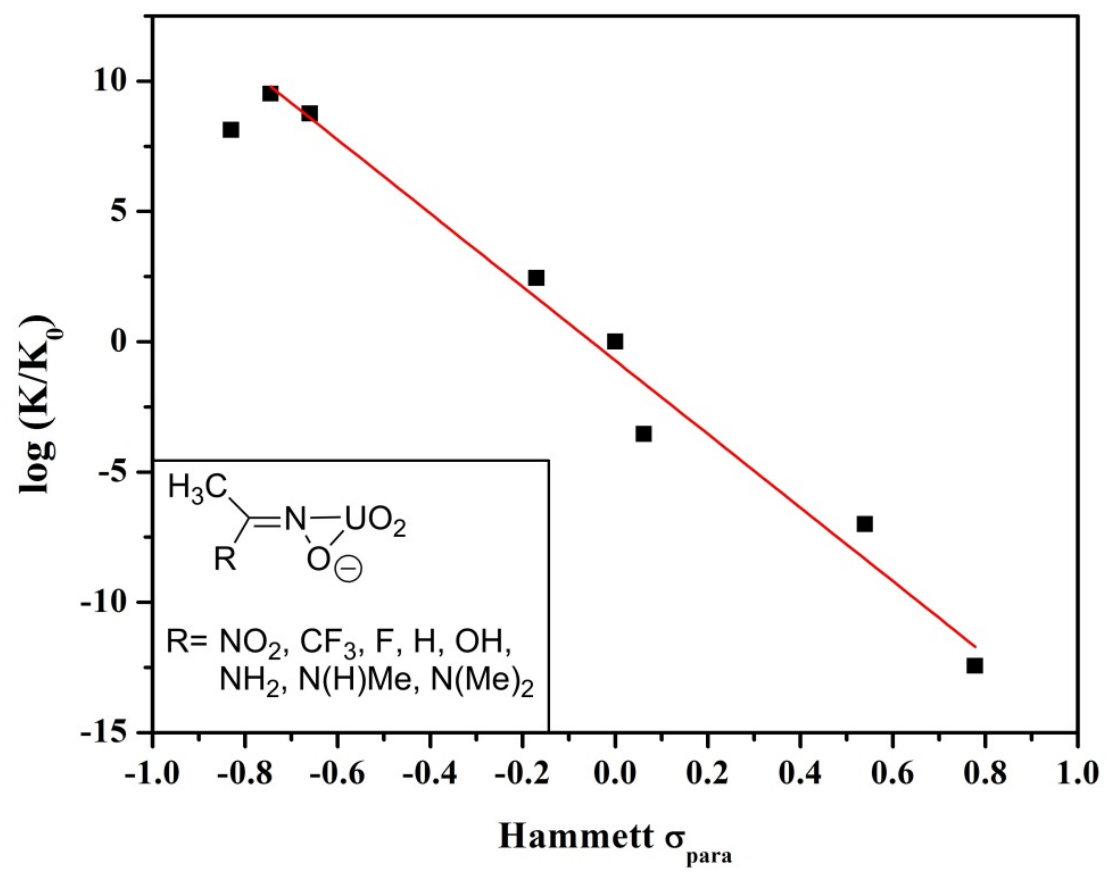

Figure 25. The plot of $\log \left(K / K_{0}\right)$ vs. Hammett $\sigma_{\text {para }}$ constants for the adjacent substituents on oximatederived ligands binding to uranyl. $K_{0}$ is determined from the uranyl-oximate complex, where $\mathrm{H}$ is the adjacent substituent. Values for $K$ were obtained from calculated values of $\Delta G$. The red line is the linear regression with $R^{2}=0.9773$. The point corresponding to the dimethyl amine substituent $\left(\mathrm{N}(\mathrm{Me})_{2}\right)$ was not included in calculating the regression due to the sterically-induced distortion in orbital conjugation. Inset: The scope of oximate-derived ligands, as bound to $\mathrm{UO}_{2}{ }^{2+}$ in an $\eta^{2}$ fashion. Three aquo ligands complete the coordination sphere for all complexes.

Observing the optimized structures of the uranyl-oximate complexes, several key bond lengths and angles are strongly influenced by the strength of the uranyl-oxime bond. A systematic weakening of $\mathrm{U}=\mathrm{O}$ bonds are observed as $\pi$-donation is increased, lengthening from $1.772 \AA$ for an adjacent nitro group to $1.789 \AA$ for dimethyl amino. The decrease in $\mathrm{U}=\mathrm{O}$ bonding can also be quantified by WBIs, with values of 2.25, 2.24, and 2.22 for uranyl complexes with nitrooximate, oximate, and amidoximate, respectively. Additionally, the $\mathrm{O}=\mathrm{U}=\mathrm{O}$ bond angle appears to generally decrease with increased electron donation, though there are several exceptions to this trend. As expected, U-O and U-N bond lengths also decrease as a function of bond strength, and thus electron donation. A summary of key bond lengths and angles is presented in Table 13. 
Table 3. Representative Bond Lengths $(\AA)$ and $U=O$ Angle $\left(^{\circ}\right)$ for Uranyl-Oximate Complexes

\begin{tabular}{|c|c|c|c|c|c|c|c|}
\hline Substituent & $\mathrm{U}=\mathrm{O}$ & $<\mathrm{U}=\mathrm{O}$ & $\mathrm{U}-\mathrm{OH}_{2}$ & $\mathrm{U}-\mathrm{O}_{\text {oxime }}$ & $\mathrm{U}-\mathrm{N}$ & $\mathrm{N}-\mathrm{O}$ & $\mathrm{N}=\mathrm{C}$ \\
\hline$-\mathrm{NO}_{2}$ & 1.772 & 176.88 & 2.458 & 2.340 & 2.440 & 1.306 & 1.284 \\
\hline$-\mathrm{CF}_{3}$ & 1.778 & 177.25 & 2.481 & 2.287 & 2.408 & 1.351 & 1.269 \\
\hline$-\mathrm{F}$ & 1.775 & 176.68 & 2.467 & 2.313 & 2.414 & 1.331 & 1.280 \\
\hline$-\mathrm{H}$ & 1.780 & 175.95 & 2.481 & 2.282 & 2.392 & 1.351 & 1.276 \\
\hline$-\mathrm{OH}$ & 1.783 & 176.05 & 2.493 & 2.269 & 2.372 & 1.363 & 1.282 \\
\hline$-\mathrm{NH}_{2}$ & 1.786 & 174.32 & 2.497 & 2.255 & 2.349 & 1.383 & 1.300 \\
\hline$-\mathrm{N}(\mathrm{H}) \mathrm{Me}$ & 1.787 & 172.92 & 2.492 & 2.252 & 2.344 & 1.387 & 1.306 \\
\hline$-\mathrm{N}(\mathrm{Me})_{2}$ & 1.789 & 173.25 & 2.498 & 2.251 & 2.339 & 1.378 & 1.309 \\
\hline
\end{tabular}

Inspection of the molecular orbitals involved in uranyl-oximate binding reveals several important interactions to be affected by the nature of the adjacent substituent. First, electron withdrawing functions lowered the energy of the MOs involved in bonding with uranyl. Substituting $-\mathrm{NO}_{2}$ for $-\mathrm{NH}_{2}$ lowered the energy of the bonding orbitals by an average $1.34 \mathrm{eV}$, corresponding with weaker bonding as a result of mismatched orbital energies. Second, the contribution from the oxime oxygen lone-pair to the uranyl $5 \mathrm{f}$ basis functions is directly related to the extent of electron donation, as can be seen by comparing orbitals for uranyl-amidoximate, uranyl-oximate, and uranyl-nitrooximate. While minor interaction can qualitatively be observed for all ligands with electron donating functions, NBO analysis suggests bonding occurs between the oxygen p-orbital and uranyl on ligands with strong electron donating groups $\left(-\mathrm{NH}_{2}\right.$, $\left.\mathrm{N}(\mathrm{H}) \mathrm{CH}_{3}, \mathrm{~N}\left(\mathrm{CH}_{3}\right)_{2}\right)$. Finally, electron donation affects the delocalization of the oximate N-O $\pi$ orbitals into those of the uranyl oxygen lone pairs. This secondary interaction, most clearly observed in orbital 44 of the uranyl-AO complex (Figure 24), can be probed by examining the WBIs for the oximate N-O interactions with the uranyl oxygen. Values of $0.0171,0.0190$, and 0.0192 were obtained for uranyloxygen bonding with nitrooximate, oximate, and amidoximate complexes, respectively.

To elucidate the relationship between electron donation and bond strength, $\Delta G$ values obtained by DFT calculation were used to calculate equilibrium constants from the following formula:

$$
\Delta G=-R T(\ln K)
$$

where $R$ is the gas constant, $T$ is temperature in Kelvin and assumed to be 298 in all calculations, and $K$ is the equilibrium constant. The oxime ligand with hydrogen in the adjacent position was used as a reference and for calculating the equilibrium constant $K_{0}$. The log of $K / K_{0}$ for each ligand was plotted against the corresponding Hammett $\sigma_{\text {para }}$ value, obtained from the literature. The Hammett $\sigma$ value is a measure of a substituent's electron donating or withdrawing abilities. While no values for $\sigma_{\text {ortho }}$ are experimentally available due to the influence of associated steric factors, values for $\sigma_{\text {para }}$ primarily account for electron donation via resonance and are an accurate substitute.

Plotting $\log (\mathrm{K} / \mathrm{K} 0)$ vs. $\sigma_{\text {para }}$ demonstrates a direct relationship between $\mathrm{UO}_{2}{ }^{2+}$ binding strength and the electron donating properties of the adjacent substituent, as shown in Figure 25 and summarized in Table 14. The linear relationship is clearly demonstrated, as a linear regression yields an $R^{2}=0.9773$. WBIs are in agreement with the thermodynamic data, yielding lower values for oximates with electron withdrawing functions as compared to those with electron donating functions. For example, the uranylnitrooxime WBI of $1.09\left(\sigma_{\text {para }}=0.78\right)$ can be compared with that of uranyl-oximate, $1.26\left(\sigma_{\text {para }}=0\right)$, and amidoxime, $1.40\left(\sigma_{\text {para }}=-0.66\right)$, showing the direct relationship between bonding and electron donation. One noteworthy exception arrives from substituting the adjacent amine $\left(\sigma_{\text {para }}=-0.66\right)$ for the stronger donating dimethyl amine $\left(\sigma_{\text {para }}=-0.83\right)$, resulting in a complex that is approximately $0.84 \mathrm{kcal} / \mathrm{mol}$ less 
stable. A structural investigation revealed that although the $\mathrm{O}-\mathrm{N}=\mathrm{C}-\mathrm{N}$ sequence was still largely coplanar, a slight dihedral distortion of approximately $3^{\circ}$ occurred due to the steric bulk of the dimethyl groups, disrupting the conjugated series of $\pi$ orbitals and decreasing the ability of the substituent to donate electron density to the oxime function. Removing one methyl group decreased the opara value for the substituent $\left(\sigma_{\text {para }}=-0.74\right.$ ) but eliminated the structural distortion. The restored hyperconjugation facilitates electron donation as demonstrated by the lowest $\Delta \mathrm{G}$ value obtained for this series, emphasizing the necessity of preserving the conjugated system of $\pi$ orbitals in the oximate-derived sorbents.

Table 14. Hammett $\sigma_{\text {para }}$ log $\left(K / K_{0}\right), \Delta G$, Wiberg Bond Index, Total Interaction Energy, Orbital Interaction Energy, and Steric Interaction Energy for Oximate Derived Ligands Binding to Uranyl

\begin{tabular}{|c|c|c|c|c|c|c|c|}
\hline Substituent & $\sigma_{\text {para }}$ & $\log \left(K / K_{0}\right)$ & $\Delta G$ & WBI & $\begin{array}{c}E_{\text {int }} \\
(\mathrm{kcal} / \mathrm{mol})\end{array}$ & $\begin{array}{c}E_{\text {orb }} \\
(\mathrm{kcal} / \mathrm{mol})\end{array}$ & $\begin{array}{c}E_{\text {steric }} \\
(\mathrm{kcal} / \mathrm{mol})\end{array}$ \\
\hline$-\mathrm{NO}_{2}$ & 0.78 & -12.45 & -27.34 & 1.09 & -35.52 & -71.99 & 36.47 \\
\hline$-\mathrm{CF}_{3}$ & 0.54 & -7.01 & -34.56 & 1.16 & -44.62 & -77.55 & 32.93 \\
\hline$-\mathrm{F}$ & 0.06 & -3.55 & -39.14 & 1.21 & -49.48 & -82.44 & 32.96 \\
\hline$-\mathrm{H}$ & 0.00 & 0.00 & -43.85 & 1.26 & -53.58 & -87.83 & 34.25 \\
\hline$-\mathrm{OH}$ & -0.17 & 2.44 & -47.08 & 1.31 & -58.25 & -92.33 & 34.08 \\
\hline$-\mathrm{NH}_{2}$ & -0.66 & 8.76 & -55.47 & 1.40 & -62.83 & -104.42 & 41.59 \\
\hline$-\mathrm{N}(\mathrm{H}) \mathrm{Me}$ & -0.75 & 9.51 & -56.45 & 1.42 & -64.38 & -100.76 & 36.38 \\
\hline$-\mathrm{N}(\mathrm{Me})_{2}$ & -0.83 & 8.13 & -54.63 & 1.44 & -68.75 & -104.01 & 35.26 \\
\hline
\end{tabular}

To investigate the ionic contribution to amidoximate-uranyl bonding, the energy decomposition analysis of Morokuma and Ziegler was performed using the AOMix software platform. Optimized uranyl-AO structures were split into two fragments to analyze the interaction between uranyl and the AO portions of the molecule. The total electronic interaction energy $\left(E_{i n t}\right)$ between fragments is the sum of electrostatic energy $\left(E_{e s}\right)$, Pauli repulsion $\left(E_{e x}\right)$, and orbital interaction energy $\left(E_{\text {orb }}\right)$ :

$$
E_{\text {int }}=E_{\text {es }}+E_{\text {ex }}+E_{\text {orb }}
$$

AOMix conbines the electrostatic energy and Pauli repulsion into a single term, $E_{\text {steric }}$. Thus,

$$
E_{\text {int }}=E_{\text {steric }}+E_{\text {orb }}
$$

The values for $E_{\text {int }}$ for all uranyl-amidoximate molecules follow the same trend as the Gibbs Free Energies calculated previously. A linear regression on the plot of $E_{\text {int }} \mathrm{vs} \sigma_{\text {para }}$ gives an $\mathrm{R}^{2}$ of 0.9652 , revealing the direct dependence on electron donation from the substituent adjacent to the oximate function. The majority of $E_{\text {int }}$ is due to contributions from the orbital interaction energy, $E_{\text {orb }}$, which range from $-71.99 \mathrm{kcal} / \mathrm{mol}\left(-\mathrm{NO}_{2}\right)$ to $-104.42\left(-\mathrm{NH}_{2}\right)$, indicative of covalent bonding interactions. While most $E_{\text {orb }}$ values obey the same pattern as Gibbs Energies and $E_{\text {int }}$ values, increasing as a function of electron donation, the methyl amine substituent $(-\mathrm{N}(\mathrm{H}) \mathrm{Me}$ ) has a lower orbital interaction energy than both AO ($\mathrm{NH}_{2}$ ) and dimethyl amine (-N(Me) $)$ by approximately $4 \mathrm{kcal} / \mathrm{mol}$ in each instance. The linear regression of $E_{\text {orb }}$ vs $\sigma_{\text {para }}$ yields an $\mathrm{R}^{2}$ of 0.9686 . Values for $E_{\text {int }}$ and $E_{\text {orb }}$ are included in Table 14.

Calculated values for $E_{\text {steric }}$ generally increase with increasing electron donation, with the lowest values obtained for the trifluoro methane substituent $\left(-\mathrm{CF}_{3}\right)$, and the highest values attributed to AO ($\mathrm{NH}_{2}$ ). Values for $E_{\text {steric }}$ are composed of the electrostatic interactions, $E_{\text {es }}$, and the Pauli repulsion energy, $E_{e x}$. For all structures investigated, the $E_{\text {steric }}$ term is positive in energy, indicating Pauli repulsion overwhelms any constructive electrostatic interactions or ionic bonding between uranyl and the ligand. 
The Pauli repulsion energy is a function of several factors, including orbital overlap which is influenced by electron donation from the substituent adjacent to the oximate moiety. Electron donation also dictates the extent of electrostatic interaction, preventing straightforward comparison between functional groups or trivial separation of electrostatic and Pauli repulsion contributions. As the difference in $E_{\text {steric }}$ values spans less than $9 \mathrm{kcal} / \mathrm{mol}$ for all complexes, the difference in ionic bonding is not expected to be a significant factor in bond strength. Values for $E_{\text {steric }}$ are displayed in Table 14.

We sought to increase the electron donation to the oxime function by anchoring it to an imidazole. While a $\sigma_{\text {para }}$ value for this group cannot be meaningfully obtained, the system of conjugated $\pi$ orbitals and two adjacent electron donors suggests strong contribution to the binding oxime function. The calculated value for the uranyl-imidazole-oximate complex confirmed the previously established trend, with a $\Delta G$ of $-60.92 \mathrm{kcal} / \mathrm{mol}$, approximately $5 \mathrm{kcal} / \mathrm{mol}$ more favorable than the traditional amidoxime function. NBO values indicate the bonding interaction between uranium and the oxime oxygen to be 8.4\% uranium in character, as compared with $7.2 \%$ for amidoxime. This suggests the added electron density from the imidazole increased the energy of the oxygen lone pair p orbital, allowing greater interaction with the $\sigma(\mathrm{f})$ orbitals on uranium and resulting in a stronger bond. This is supported by the larger bond index of 1.4962 in conjunction with $\mathrm{U}=\mathrm{O}$ bond lengths increasing to $1.794 \AA$ and $\mathrm{U}-\mathrm{O}$ and $\mathrm{U}$ $\mathrm{N}$ bond lengths decreasing to $2.237 \AA$ and $2.350 \AA$, respectively.

Unlike the uranyl-oximate complexes discussed above, uranyl-imidazole-oximate adopts an optimized geometry where the aromatic imidazole is oriented out of the uranyl equatorial plane (Figure 26). This configuration allows for an increase in overlap between the oxygen and nitrogen lone pairs with the uranyl $1 \delta_{\mathrm{u}}$, as well as improving overlap for the $\pi(\mathrm{O}-\mathrm{N})$ binding with the uranyl $1 \pi_{\mathrm{g}}$. This yields NBO values of 0.0092 and 0.0103 for bonding between the uranyl oxygen and the oxime nitrogen and oxygen, respectively, confirming this improved orbital interaction as compared to the previous oxime-derived ligands. Additionally, AOMix calculations for this molecule yielded the highest values for $E_{\text {int }}(-73.52$ $\mathrm{kcal} / \mathrm{mol})$ and $E_{\text {orb }}(-113.54 \mathrm{kcal} / \mathrm{mol})$ of all ligands investigated.
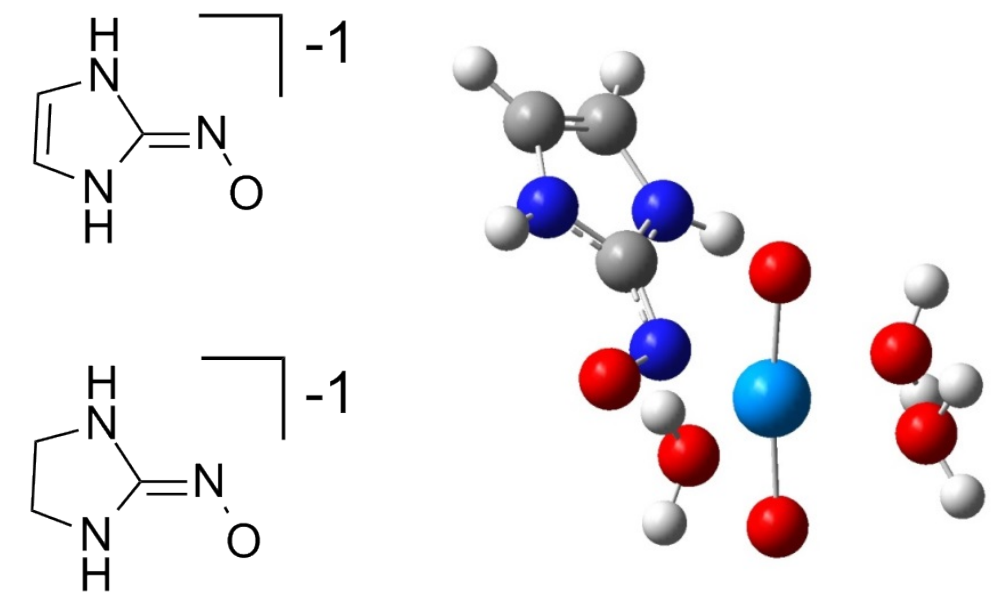

Figure 26. Left) Imidazole-oximate (top) and imidazolidin-oximate (bottom). (Right) Optimized structure for uranyl-imidazole-oximate.

While the thermodynamics of the imidazole-oximate ligand were encouraging, stability considerations preclude this particular ligand for use extracting uranyl from seawater. The class of 2hydroxylaminoimidazoles is somewhat stable when protonated under acidic conditions, but is observed to 
decompose rapidly at neutral or alkaline $\mathrm{pH}$. It is proposed nucleophilic hydroxide ions add to the imidazole double bond, forming an iminoimidazolidine diol while releasing the oxime $\mathrm{O}$ as a hydroxyl. Substitution of imidazole with benzimidizole would presumably stabilize the ligand, as has been demonstrated previously with cyclic imide dioximes, but DFT calculations reveal weaker bonding with uranyl than amidoxime by approximately $2 \mathrm{kcal} / \mathrm{mol}$. A similar phenomenon has been observed in potentiometric and spectrophotometric studies with cyclic imidedioximes binding to uranyl, which can likely be attributed to electron withdrawing from the fused benzene ring.

Reducing the imidazole-oximate to an imidazolidin-oximate provides an alternative method to increase the stability under environmental conditions (Figure 26). Though the system of $\pi$ orbitals conjugated throughout the imidazole material is not preserved, two adjacent nitrogen provide strong electron donation to the oximate moiety. The resulting $\Delta G$ for uranyl-imidazolidin-oximate complex formation is $-58.06 \mathrm{kcal} / \mathrm{mol}$, roughly $3 \mathrm{kcal} / \mathrm{mol}$ lower in energy than the analogous amidoxime complex, but more than $2 \mathrm{kcal} / \mathrm{mol}$ higher than the unstable imidazole variant. Like the uranyl-imidazole-oximate complex, the imidazolidin-oximate coordinates out of the equatorial plane of uranyl, with a WBI only slightly lower than the imidazole complex, and a similar amount of uranium character in the oxime oxygen lone-pair interaction with the uranium $5 f$ basis orbitals. AOMix calculations yielded similar $E_{\text {int }}$ $(-69.29 \mathrm{kcal} / \mathrm{mol}), E_{\text {orb }}(-108.93 \mathrm{kcal} / \mathrm{mol})$, and $E_{\text {steric }}(39.64 \mathrm{kcal} / \mathrm{mol})$ values compared to the uranylimidazole molecule. Due to the improved stability and electron donation, this imidazole-oximate ligand is expected to be highly competitive for binding uranyl in environmental conditions.

\section{Identification of promising nanostructured sorbents for further study}

Several of the most promising nanostructured sorbents were selected for additional study. While mesoporous silica materials showed impressive uranium sorption, most can be attributed to nonspecific physorption, making their continued use undesirable. Furthermore, their instability under environmental conditions precludes their use in seawater studies. However, the robust mesoporous carbon nanoparticles show no sorptive properties prior to functionalization, and are stable under a wide range of harsh conditions. Furthermore, advanced processing techniques allow the fabrication of open cell carbon foams and other monoliths, facilitating large scale functionalization and seawater testing. Continued research using this platform is ongoing.

Metal-organic frameworks showed remarkable uranium sorption due to the cooperative binding interactions in conjunction with their ultra-high porosity and low density. Work is currently underway to facilitate their deployment in marine tests, both by increasing their stability and by preparing modes for deployment, such as grafting onto porous carbon foam.

\section{Collaborate to study extraction from seawater $w /$ nanocomposites}

Numerous samples have been sent to national laboratory collaborators for validation of reported sorption data as well as for more advanced testing. Samples of functionalized mesoporous carbon materials were sent to Pacific Northwest National Laboratory for marine toxicity study, while samples of organo-functionalized carbons were sent to Oak Ridge National Laboratory for preliminary seawater extraction studies.

We are presently working to develop materials into nanocomposites for flow-through seawater testing at Pacific Northwest National Laboratory. Their current engineering does not accommodate testing of our nanomaterials with seawater via flow-through methods. Work is ongoing to develop monolithic materials or monolith-functionalized materials to permit these advanced studies. 
NEUP 11-3151

\section{LIST OF ANY PUBLICATION OR INFORMATION RELEASE MADE OF MATERIAL DEVELOPED OR MAINTAINED THROUGH THE PERFORMANCE OF THE CONTRACT}

Vivero-Escoto, J. L.; Carboni, M.; Abney, C.W.; deKrafft, K.E.; Lin, W. "Organo-functionalized Mesoporous Silicas for Efficient Uranium Extraction.” Microporous Mesoporous Mater. 2013, 180, 22.

Carboni, M.; Abney, C.W.; Liu, S.; Lin, W. "Highly Porous and Stable Metal-organic Frameworks for Uranium Extraction.” Chem. Sci., 2013, 4, 2396.

Carboni, M.; Abney, C.W.; Tayor-Pashow, K.M.; Vivero-Escoto, J.L.; Lin, W. "Uranium Sorption with Functionalized Mesoporous Carbon Materials.” Ind. Eng. Chem. Res. 2013, 52, 15187.

Abney, C.W.; Liu, S.; Lin, W. “Tuning Amidoximate to Enhance Uranyl Binding: A Density Functional Theory Study.” J. Phys. Chem. A. 2013, 117, 11558. 